\title{
Polynomially Bounding the Number of Minimal Separators in Graphs: Reductions, Sufficient Conditions, and a Dichotomy Theorem
}

\author{
Martin Milanič ${ }^{*} \quad$ Nevena Pivač $\check{c}^{\dagger}$ \\ University of Primorska, IAM, Muzejski trg 2, SI-6000 Koper, Slovenia \\ University of Primorska, FAMNIT, Glagoljaška 8, SI-6000 Koper, Slovenia \\ martin.milanic@upr.si, nevena.pivac@iam.upr.si \\ Submitted: Mar 10, 2020; Accepted: Jan 25, 2021; Published: Feb 26, 2021 \\ (C) The authors. Released under the CC BY-ND license (International 4.0).
}

\begin{abstract}
A graph class is said to be tame if graphs in the class have a polynomially bounded number of minimal separators. Tame graph classes have good algorithmic properties, which follow, for example, from an algorithmic metatheorem of Fomin, Todinca, and Villanger from 2015. We show that a hereditary graph class $\mathcal{G}$ is tame if and only if the subclass consisting of graphs in $\mathcal{G}$ without clique cutsets is tame. This result and Ramsey's theorem lead to several types of sufficient conditions for a graph class to be tame. In particular, we show that any hereditary class of graphs of bounded clique cover number that excludes some complete prism is tame, where a complete prism is the Cartesian product of a complete graph with a $K_{2}$. We apply these results, combined with constructions of graphs with exponentially many minimal separators, to develop a dichotomy theorem separating tame from non-tame graph classes within the family of graph classes defined by sets of forbidden induced subgraphs with at most four vertices.
\end{abstract}

Mathematics Subject Classifications: 05C40, 05C75, 05C30, 05C76, 05C69

\section{Introduction}

The main concept studied in this paper is that of a minimal separator in a graph. Given a graph $G$, a minimal separator in $G$ is a set of vertices that separates some non-adjacent

\footnotetext{
* Supported in part by the Slovenian Research Agency (I0-0035, research program P1-0285, and research projects J1-9110, N1-0102, and N1-0160). Part of the work was done while the author was visiting Osaka Prefecture University in Japan, under the operation Mobility of Slovene higher education teachers 2018-2021, co-financed by the Republic of Slovenia and the European Union under the European Social Fund.

†Supported in part by the Slovenian Research Agency Agency (research program P1-0285, research projects N1-0102, J1-9110, and a Young Researchers Grant).
} 
vertex pair $a, b$ and is inclusion-minimal with respect to this property. Minimal separators in graphs are important for sparse matrix computations, via their connection with minimal triangulations (see [27] for a survey). Many graph algorithms and characterizations are based on minimal separators (see, e.g., $[2,5,7-9,11,16,17,21,32,41,50]$ ).

In this work we focus on graphs with "few" minimal separators. Such graphs enjoy good algorithmic properties. Many problems that are NP-hard for general graphs become polynomial-time solvable for classes of graphs with a polynomially bounded number of minimal separators. This is the case for TrEewidTh and Minimum Fill-In [12], for Maximum Independent Set, Feedback Vertex Set, and more generally the problem of finding a maximum induced subgraph of treewidth at most a constant $t$ [23], and for Distance- $d$ Independent SET for even $d$ [38]. The result of Fomin and Villanger from [23] was further generalized in 2015 by Fomin, Todinca, and Villanger [22] to an algorithmic metatheorem concerning induced subgraphs with properties expressible in a certain logical system. Their approach captures many problems including MAXIMUM Induced Matching, Longest Induced Path, Maximum Induced Subgraph With no Cycles of Length 0 Modulo $m$ where $m$ is any fixed positive integer, and Maximum $\mathcal{F}$-Minor-Free Induced Subgraph where $\mathcal{F}$ is any set of graphs containing a planar graph.

All these results make it important to identify classes of graphs with a polynomially bounded number of minimal separators. Known classes with this property include chordal graphs [46] and their generalization weakly chordal graphs [11], permutation graphs [6, 29] and more generally cocomparability graphs of bounded interval dimension [20], circular-arc graphs [33], circle graphs [30], polygon circle graphs [49], distance-hereditary graphs [31], probe interval graphs [15], AT-free co-AT-free graphs [34], $P_{4}$-sparse graphs [39], extended $P_{4}$-laden graphs [42], and graphs with minimal separators of bounded size [48]. Moreover, it is known that a class of graphs has a polynomially bounded number of minimal separators if and only if it has a polynomially bounded number of potential maximal cliques [12].

This brings us to the central property of a graph class studied in this paper. We say that a graph class is tame if graphs in the class have a polynomially bounded number of minimal separators. A precise definition requires some notation. Given two non-adjacent vertices $a$ and $b$ in a graph $G$, a set $S \subseteq V(G) \backslash\{a, b\}$ is an $a, b$-separator if $a$ and $b$ are contained in different connected components of $G-S$. If $S$ contains no other $a, b$-separator as a proper subset, then $S$ is a minimal $a, b$-separator. We denote by $\mathcal{S}_{G}(a, b)$ the set of all minimal $a, b$-separators. A minimal separator in $G$ is a set $S \subseteq V(G)$ that is a minimal $a, b$-separator for some pair of non-adjacent vertices $a$ and $b$. We denote by $\mathcal{S}_{G}$ the set of all minimal separators in $G$ and by $s(G)$ the cardinality of $\mathcal{S}_{G}$.

Definition 1. We say that a graph class $\mathcal{G}$ is tame if there exists a polynomial $p: \mathbb{R} \rightarrow \mathbb{R}$ such that for every graph $G \in \mathcal{G}$, we have $s(G) \leqslant p(|V(G)|)$.

The main purpose of this paper is to further the knowledge of tame graph classes, and we do this from three interrelated points of view. 


\subsection{Operations preserving tame graph classes}

The problem of computing the number of minimal separators of a disconnected graph can be reduced to the same problem on each component, and similarly for graphs whose complements are disconnected (see, e.g., [42]). We examine the consequences of these results for tame graph classes and, as our main result regarding operations preserving tame graph classes, show that the problem of determining if a hereditary graph class $\mathcal{G}$ is tame can be reduced to the same problem on the subclass consisting of the graphs in $\mathcal{G}$ that have no clique cutsets. A clique cutset in a graph $G$ is a clique $C$ in $G$ such that the graph $G-C$ is disconnected. A graph is said to be an atom if it has no clique cutset. Given a class $\mathcal{G}$ of graphs, we denote by $A(\mathcal{G})$ the class of all atoms that are induced subgraphs of a graph in $\mathcal{G}$.

Theorem 2. For every graph class $\mathcal{G}$, if $A(\mathcal{G})$ is tame, then so is $\mathcal{G}$.

\subsection{Sufficient conditions}

We identify several sufficient conditions for a graph class to be tame. Each condition reveals an infinite family of tame graph classes.

To state the first one, we need some definitions. Given a family $\mathcal{F}$ of graphs, we say that a graph $G$ is $\mathcal{F}$-free if no induced subgraph of $G$ is isomorphic to a member of $\mathcal{F}$. A graph class $\mathcal{G}$ is hereditary if it is closed under vertex deletion, or, equivalently, if there exists a set $\mathcal{F}$ of graphs such that $\mathcal{G}$ is exactly the class of $\mathcal{F}$-free graphs. A complete prism of order $n$ is the Cartesian product of $K_{n}$ with a $K_{2}$, that is, a graph consisting of two disjoint copies of the $n$-vertex complete graph and a perfect matching between them. The clique cover number of a graph $G$ is the smallest number of pairwise disjoint cliques in $G$ whose union is the vertex set of $G$. Note that every complete prism of order at least two has clique cover number two. As we will explain in Section 5, the class of complete prisms is not tame. This implies that for every $k \geqslant 2$, the class of graphs of clique cover number at most $k$ is not tame. Furthermore, the class of $C_{4}$-free graphs is not tame (cf. Corollary 41), which implies that for every $n \geqslant 2$, the class of all graphs not containing an induced subgraph isomorphic to the complete prism of order $n$ is not tame. Our first sufficient condition states that for hereditary graph classes, combining the two properties of bounding the clique cover number and excluding some complete prism results in a tame graph class.

Theorem 3. Every hereditary class of graphs of bounded clique cover number that does not contain all complete prisms is tame.

The second sufficient condition is derived from the first one and is about subclasses of $C_{4}$-free graphs.

Theorem 4. For every positive integer $k$, the class of $\left\{P_{2}+k P_{1}, C_{4}\right\}$-free graphs is tame.

The third sufficient condition is proved using Ramsey's theorem. 
Theorem 5. For every two positive integers $k$ and $\ell$, the class of $\left\{P_{2}+k P_{1}, K_{\ell}+P_{2}\right\}$-free graphs is tame.

In some sense, these results are best possible. The class of all complete prisms (and consequently the class of all $\left(P_{2}+2 P_{1}\right)$-free graphs) is not tame, while the class of elementary walls shows that the class of $\left(K_{3}+P_{2}\right)$-free graphs is not tame. (See Section 5 for details.)

\subsection{A dichotomy result}

We use the above results, along with constructions of graphs with exponentially many minimal separators, to completely characterize which graph classes defined by forbidden induced subgraphs with at most four vertices are tame. To describe the result, we need to introduce some notation. Given two families $\mathcal{F}$ and $\mathcal{F}^{\prime}$ of graphs, we write $\mathcal{F} \unlhd \mathcal{F}^{\prime}$ if the class of $\mathcal{F}$-free graphs is contained in the class of $\mathcal{F}^{\prime}$-free graphs, or, equivalently, if every $\mathcal{F}$-free graph is also $\mathcal{F}^{\prime}$-free. It is well known and not difficult to see that relation $\mathcal{F} \unlhd \mathcal{F}^{\prime}$ can be checked by means of the following criterion, which becomes particularly simple for finite families $\mathcal{F}$ and $\mathcal{F}^{\prime}$.

Observation 6. For every two graph families $\mathcal{F}$ and $\mathcal{F}^{\prime}$, we have $\mathcal{F} \unlhd \mathcal{F}^{\prime}$ if and only if every graph from $\mathcal{F}^{\prime}$ contains an induced subgraph isomorphic to a member of $\mathcal{F}$.

Some small graphs are named as in Fig. 1.

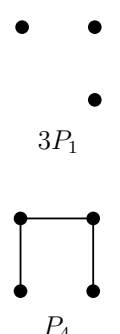

$P_{4}$
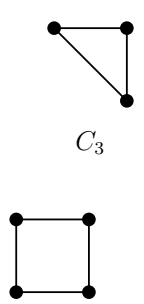

$C_{4}$
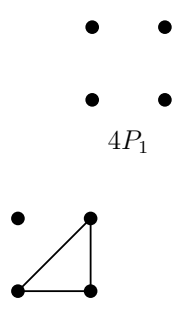

$C_{3}+P_{1}$
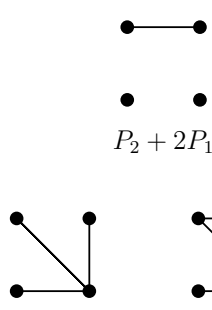

claw
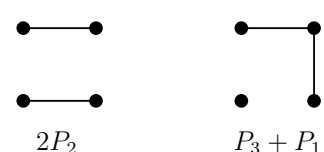

$P_{3}+P_{1}$

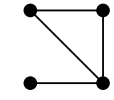

paw

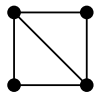

diamond

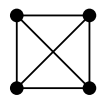

$K_{4}$

Figure 1: Some graphs on at most 4 vertices.

The characterization is as follows.

Theorem 7. For every family $\mathcal{F}$ of graphs with at most 4 vertices, the following statements are equivalent.

1. The class of $\mathcal{F}$-free graphs is tame.

2. The class of $\mathcal{F}$-free graphs does not contain any of the following graph classes: the class of $\left\{C_{3}, C_{4}\right\}$-free graphs, the class of $\left\{3 P_{1}\right.$, diamond $\}$-free graphs, and the class of $\left\{\right.$ claw, $K_{4}, C_{4}$, diamond $\}$-free graphs.

3. $\mathcal{F} \unlhd \mathcal{F}^{\prime}$ for at least one of the following families $\mathcal{F}^{\prime}$ : 


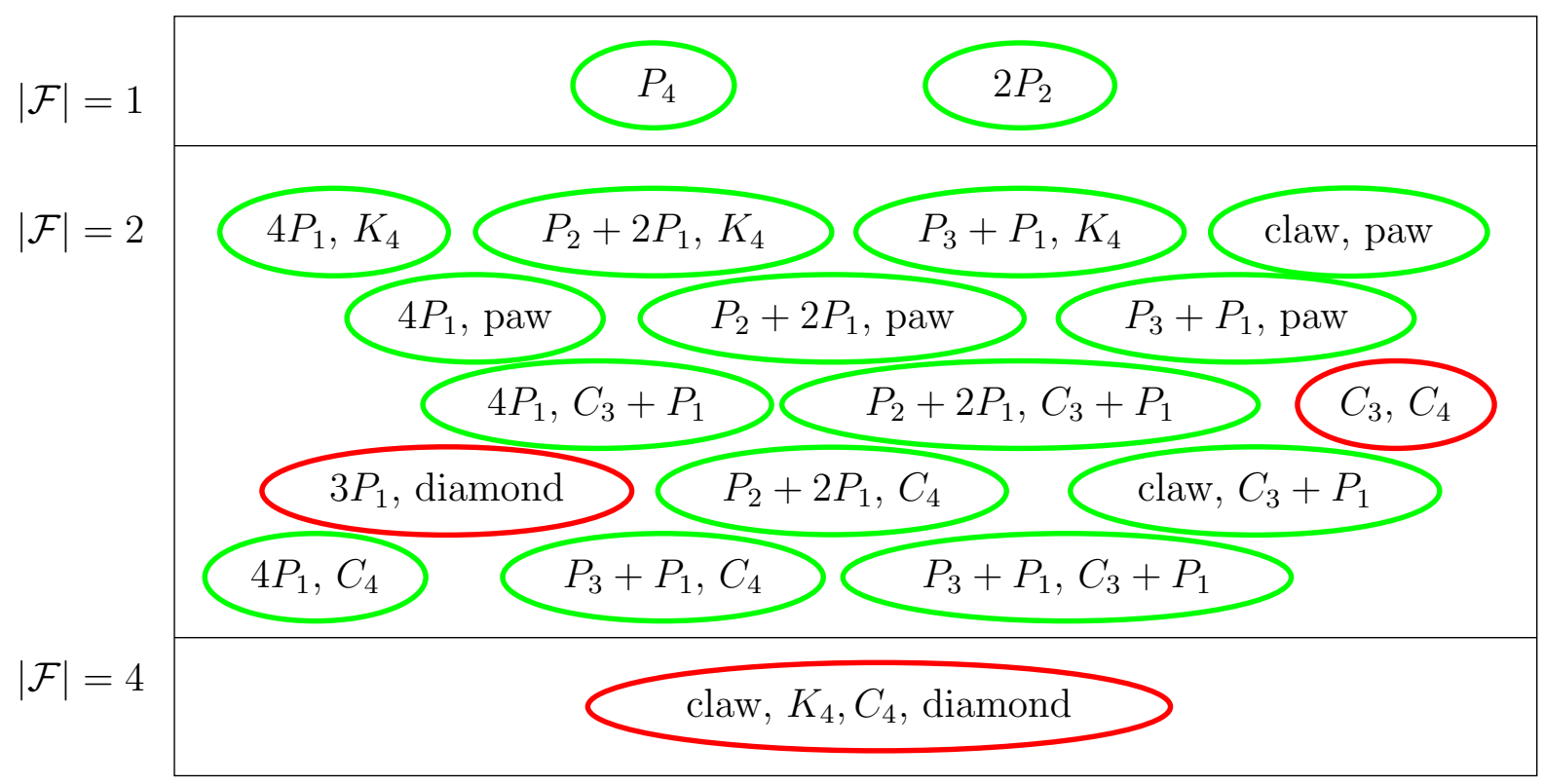

Figure 2: Overview of the dichotomy result. Maximal tame classes correspond to sets $\mathcal{F}$ of forbidden induced subgraphs depicted in green ellipses, while minimal non-tame classes correspond to sets depicted in red ellipses (in brighter, resp., darker ellipses in gray-scale printing).
i) $\mathcal{F}^{\prime}=\left\{P_{4}\right\}$,
ii) $\mathcal{F}^{\prime}=\left\{2 P_{2}\right\}$,
iii) $\mathcal{F}^{\prime}=\{F$, paw $\}$ for some $F \in\left\{4 P_{1}, P_{2}+2 P_{1}, P_{3}+P_{1}\right.$, claw $\}$,
iv) $\mathcal{F}^{\prime}=\left\{F, C_{3}+P_{1}\right\}$ for some $F \in\left\{4 P_{1}, P_{2}+2 P_{1}, P_{3}+P_{1}\right.$, claw $\}$,
v) $\mathcal{F}^{\prime}=\left\{F, K_{4}\right\}$ for some $F \in\left\{4 P_{1}, P_{2}+2 P_{1}, P_{3}+P_{1}\right\}$,
vi) $\mathcal{F}^{\prime}=\left\{F, C_{4}\right\}$ for some $F \in\left\{4 P_{1}, P_{2}+2 P_{1}, P_{3}+P_{1}\right\}$.

To appreciate Theorem 7, note that up to isomorphism, there are 11 four-vertex graphs, which means that there are $2^{11}=2048$ different graph classes defined by a set of forbidden induced subgraphs with exactly four vertices, and even more graph classes defined by a set of forbidden induced subgraphs with at most four vertices.

In Fig. 2 we give an overview of maximal tame and minimal non-tame classes of $\mathcal{F}$-free graphs, where $\mathcal{F}$ contains graphs with at most four vertices. A similar figure with respect to boundedness of the clique-width can be found in [13].

Related work. To the best of our knowledge, the results of this paper represent the first set of results towards a systematic study of the problem of classifying hereditary graph classes with respect to the existence of a polynomial bound on the number of minimal separators. Dichotomy studies for many other problems in mathematics 
and computer science are available in the literature in general, as well as within the field of graph theory. This includes dichotomy studies related to boundedness of the clique-width [19], boundedness of the chromatic number of digraphs [4], price of connectivity and independence $[18,26]$, and computational complexity of a number of algorithmic problems such as Graph Homomorphism [28], Graph Isomorphism [47], Dominating SET [36], and various coloring [24] and packing problems [14,35].

Summary of techniques. Our approach combines a variety of tools and techniques, including applications of Ramsey's theorem, structural characterizations of graphs in hereditary classes, constructions of graph families with exponentially many minimal separators, and connections between minimal separators and maximal cliques.

Structure of the paper. We collect the main notations, definitions, and some preliminary results in Section 2. In Section 3, we study the effect of various graph operations on the number of minimal separators and examine their consequences for tame graph classes, including Theorem 2. In Section 4 we prove Theorems 3-5. In Section 5 we give constructions of graphs with exponentially many minimal separators and derive several necessary conditions for a graph class to be tame. In Section 6 we prove Theorem 7 . We conclude the paper with some open problems in Section 7.

This paper is an extended version of the conference publication [37].

\section{Preliminaries}

All graphs in this paper will be finite, simple, undirected, and will have at least one vertex. We denote by $V(G)$ the vertex set of a graph $G$ and by $E(G)$ the edge set of $G$. The complement of a graph $G$ is the graph $\bar{G}$ with vertex set $V(\bar{G})=V(G)$ and edge set $E(\bar{G})=\{u v \mid u \neq v$ and $u v \notin E(G)\}$. The neighborhood of vertex $v$ in $G$ is the set of all vertices adjacent to $v$ in $G$ and is denoted by $N_{G}(v)$ (or simply $N(v)$ if $G$ is clear from the context); its elements are the neighbors of $v$ in $G$. The closed neighborhood of $v$ is defined as $N_{G}[v]=N_{G}(v) \cup\{v\}$. The degree of vertex $v$ in $G$ is defined as the number of neighbors of $v$ in $G$. We define the neighborhood of a set $X \subseteq V(G)$ as $N_{G}(X)=\left(\cup_{v \in X} N_{G}(v)\right) \backslash X$. A clique in a graph $G$ is a set of pairwise adjacent vertices. A clique cover of a graph $G$ is a partition of its vertex set into cliques. A clique is maximal if it is not contained in any other clique. An independent set in $G$ is a set of pairwise non-adjacent vertices. The independence number of $G$ is defined as the maximum size of an independent set in $G$ and denoted by $\alpha(G)$. An anticomponent of a graph $G$ is the subgraph of $G$ induced by the vertex set of a component of $\bar{G}$. A graph is anticonnected if its complement is connected.

A graph $F$ is an induced subgraph of a graph $G$ if $V(F) \subseteq V(G)$ and $E(F)=\{u v \in$ $E(G) \mid\{u, v\} \subseteq V(F)\}$; we denote this relation by $F \subseteq_{i} G$. In this case, graph $F$ will also be called the subgraph of $G$ induced by $V(F)$ and denoted by $G[V(F)]$. Given a set $S \subseteq V(G)$, we denote by $G-S$ the subgraph of $G$ induced by $V(G) \backslash S$. The fact that two graphs $G$ and $H$ are isomorphic to each other will be denoted by $G \cong H$. If $F$ and $G$ are graphs such that no induced subgraph of $G$ is isomorphic to $F$, then we say that $G$ is $F$-free. 
If $G$ is a graph and $A$ and $B$ are disjoint subsets of $V(G)$, we say that they are complete (resp., anticomplete) to each other in $G$ if $\{a b \mid a \in A, b \in B\} \subseteq E(G)$ (resp., $\{a b \mid a \in A, b \in B\} \cap E(G)=\emptyset)$. If the vertex set of $G$ can be partitioned into sets $V_{1}$ and $V_{2}$ that are anticomplete to each other in $G$, then $G$ is said to be the disjoint union of graphs $G\left[V_{1}\right]$ and $G\left[V_{2}\right]$; we denote this by $G=G\left[V_{1}\right]+G\left[V_{2}\right]$. Similarly, if the vertex set of a graph $G$ can be partitioned into two sets $V_{1}$ and $V_{2}$ that are complete to each other in $G$, we say that $G$ is the join of the subgraphs of $G$ induced by $V_{1}$ and $V_{2}$; we denote this by $G=G\left[V_{1}\right] * G\left[V_{2}\right]$. Given a non-negative integer $k$, the disjoint union of $k$ copies of $G$ is denoted by $k G$.

We denote by $P_{n}, C_{n}, K_{n}$ the path, the cycle, and the complete graph with $n$ vertices, respectively. For positive integers $m, n$, we denote by $K_{m, n}$ the complete bipartite graph with $m$ and $n$ vertices in the two parts of the bipartition. The claw is the graph $K_{1,3}$, the diamond is the graph $K_{4}$ minus an edge, and the paw is the graph $\overline{P_{3}+P_{1}}$. The girth of a graph $G$ is the smallest integer $k$ such that $C_{k}$ is a subgraph of $G$ (or $\infty$ if $G$ is acyclic). Given a graph $G$, its line graph is the graph $L(G)$ with vertex set $E(G)$ in which two distinct vertices $e$ and $e^{\prime}$ are adjacent if and only if $e$ and $e^{\prime}$ have a common endpoint as edges in $G$.

Some of our proofs will make use of the following classical result due to Ramsey [45]. Ramsey's Theorem. For every two positive integers $k$ and $\ell$, there exists a least positive integer $R(k, \ell)$ such that every graph with at least $R(k, \ell)$ vertices contains either a clique of size $k$ or an independent set of size $\ell$.

We will also need the following result from the literature, describing the structure of paw-free graphs. A graph $G$ is complete multipartite if its vertex set can be partitioned into any number of parts such that two vertices are adjacent if and only if they belong to different parts.

Theorem 8 (Olariu [40]). A connected paw-free graph $G$ is either $C_{3}$-free or complete multipartite.

Given a graph $G$ and a set $S \subseteq V(G)$, a component $C$ of the graph $G-S$ is $S$-full if every vertex in $S$ has a neighbor in $C$, or, equivalently, if $N_{G}(V(C))=S$. The following well-known lemma characterizes minimal separators (see, e.g., [25]).

Lemma 9. Given a graph $G=(V, E)$, a set $S \subseteq V$ is a minimal separator in $G$ if and only if the graph $G-S$ contains at least two $S$-full components.

Corollary 10. Let $S$ be a minimal separator in a graph $G$. Then for every $v \in S$ the set $S \backslash\{v\}$ is a minimal separator in $G-v$.

Proof. Let $G^{\prime}=G-v$ and $S^{\prime}=S \backslash\{v\}$. Since $S$ is a minimal separator in $G$, there exist two $S$-full components $C$ and $D$ in $G-S$. Since $G-S=G^{\prime}-S^{\prime}$ and $S^{\prime} \subseteq S$, it follows that $C$ and $D$ are also $S^{\prime}$-full components of $G^{\prime}-S^{\prime}$. Hence, $S^{\prime}$ is a minimal separator in $G^{\prime}$.

Corollary 11. Let $S$ be a minimal separator in a graph $G$. Then for every $S^{*} \subseteq S$ the set $S \backslash S^{*}$ is a minimal separator in $G-S^{*}$. 
The next lemma gives a necessary condition for a minimal separator in a $2 P_{2}$-free graph.

Lemma 12. Let $G$ be a $2 P_{2}$-free graph and let $S$ be a minimal separator in $G$. Then there exists a vertex $v \in V(G)$ such that $S=N(v)$.

Proof. By Lemma 9, graph $G-S$ has two $S$-full components $C$ and $D$. If both $C$ and $D$ have at least two vertices, then each of them contains at least one edge. Since $C$ and $D$ are anticomplete to each other, these edges form a $2 P_{2}$ in $G$. We may thus assume, by symmetry, that $C=\{v\}$ for some $v \in V(G)$. Then it follows that $N(v) \subseteq S$ and since every vertex of $S$ is adjacent to $v$, we must have $S=N(v)$, as claimed.

Corollary 13. The class of $2 P_{2}$-free graphs is tame.

We conclude this section with two straightforward observations about tame graph classes.

Observation 14. Let $\mathcal{G}_{1}$ and $\mathcal{G}_{2}$ be two graph classes such that $\mathcal{G}_{1} \subseteq \mathcal{G}_{2}$. If $\mathcal{G}_{2}$ is tame, then so is $\mathcal{G}_{1}$.

Definition 15. Given a non-negative integer $k$, we say that a graph class $\mathcal{G}$ is $k$-tame if $s(G) \leqslant|V(G)|^{k}-1$ for every graph $G \in \mathcal{G}$.

The reasons for including the term ' -1 ' in the definition of $k$-tame graph classes is purely technical, as it simplifies some of the statements and proofs (for example, those of Lemma 24).

Lemma 16. A graph class $\mathcal{G}$ is tame if and only if it is $k$-tame for some non-negative integer $k$.

Proof. Sufficiency is trivial. To prove necessity, let $\mathcal{G}$ be a tame graph class and let $p(x)=\sum_{i=0}^{d} a_{i} x^{i}$ be a polynomial such that $s(G) \leqslant p(|V(G)|)$ for all $G \in \mathcal{G}$. We may assume that $a_{i} \geqslant 0$ for all $i$, since otherwise we may delete the terms of $p$ with negative coefficients to obtain a polynomial $q$ such that $s(G) \leqslant q(|V(G)|)$ for all $G \in \mathcal{G}$. Moreover, we may assume that $a_{0}=\ldots=a_{d}$, since otherwise, as long as there is a pair $(i, j)$ with $0 \leqslant i<j \leqslant d$ and $a_{i}<a_{j}$, we may increase the $i$-th coefficient from $a_{i}$ to $a_{j}$ to obtain a polynomial $q$ such that $s(G) \leqslant q(|V(G)|)$ for all $G \in \mathcal{G}$. Let $a$ be this common value, that is, $a_{0}=\ldots=a_{d}=a$. We thus have $p(x)=a\left(\sum_{i=0}^{d} x^{i}\right)$ and hence $p(n) \leqslant a n^{d+1}$ holds for all $n \geqslant 2$. Let $\ell$ be the least non-negative integer such that $a \leqslant 2^{\ell}$. Then, for all $n \geqslant 2$, we have $a n^{d+1} \leqslant 2^{\ell} \cdot n^{d+1} \leqslant n^{\ell} \cdot n^{d+1}=n^{d+\ell+1} \leqslant n^{d+\ell+2}-1$. Since the 1-vertex graph has no minimal separators, it follows that all $G \in \mathcal{G}$ satisfy $s(G) \leqslant|V(G)|^{d+\ell+2}-1$. Thus, taking $k=d+\ell+2$, necessity is proved.

An obvious but useful consequence of Lemma 16 is the fact that any union of finitely many tame graph classes is tame. 


\section{Graph operations}

In this section, we study the effect of various graph operations on the number of minimal separators and examine their consequences for tame graph classes. The family of minimal separators of a disconnected graph can be computed from the families of minimal separators of its components, and a similar statement holds for graphs whose complements are disconnected. The correspondences are as follows, see Pedrotti and de Mello [42].

Theorem 17. If $G$ is a disconnected graph, with components $G_{1}, \ldots, G_{k}$, then $\mathcal{S}_{G}=\{\emptyset\} \cup \bigcup_{i=1}^{k} \mathcal{S}_{G_{i}}$. If $G$ is the join of graphs $G_{1}, \ldots, G_{k}$, then $S \in \mathcal{S}_{G}$ if and only if there exists some $i \in\{1, \ldots, k\}$ and some $S_{i} \in \mathcal{S}_{G_{i}}$ such that $S=S_{i} \cup\left(V(G) \backslash V\left(G_{i}\right)\right)$.

Using this theorem we can derive the formulas for the number of minimal separators and their corollaries.

Corollary 18. Let $G$ be a disconnected graph, with components $G_{1}, \ldots, G_{k}$. Then $s(G)=$ $\sum_{i=1}^{k} s\left(G_{i}\right)+1$.

Proof. Immediate from the first statement of Theorem 17 and the fact that sets $\{\emptyset\}, \mathcal{S}_{G_{1}}, \ldots, \mathcal{S}_{G_{k}}, i \in\{1, \ldots, k\}$ are pairwise disjoint.

Corollary 19. Let $\mathcal{G}$ be a hereditary graph class and let $\mathcal{G}^{\prime}$ be the class of connected graphs in $\mathcal{G}$. Then $\mathcal{G}$ is tame if and only if $\mathcal{G}^{\prime}$ is tame.

Proof. Since $\mathcal{G}$ is hereditary, we have $\mathcal{G}^{\prime} \subseteq \mathcal{G}$. Hence, if $\mathcal{G}$ is tame, then so is $\mathcal{G}^{\prime}$ by Observation 14. Suppose that $\mathcal{G}^{\prime}$ is tame. By Lemma 16, there exists a positive integer $k$ such that $s(G) \leqslant|V(G)|^{k}-1$ for all $G \in \mathcal{G}^{\prime}$. Let $G \in \mathcal{G} \backslash \mathcal{G}^{\prime}$ and let $G_{1}, \ldots, G_{p}$ (with $p \geqslant 2$ ) be the components of $G$. Since $G$ is disconnected and for all $i \in\{1, \ldots, p\}$ we have $G_{i} \in \mathcal{G}^{\prime}$, we infer using Corollary 18 that $s(G)=\sum_{i=1}^{p} s\left(G_{i}\right)+1 \leqslant \sum_{i=1}^{p}\left(\left|V\left(G_{i}\right)\right|^{k}-1\right)+1 \leqslant$ $\sum_{i=1}^{p}\left|V\left(G_{i}\right)\right|^{k}-1 \leqslant\left(\sum_{i=1}^{p}\left|V\left(G_{i}\right)\right|\right)^{k}-1=|V(G)|^{k}-1$. It follows that $\mathcal{G}$ is tame.

Note that the above proof also shows that for every positive integer $k$, the class $\mathcal{G}$ is $k$-tame if and only if $\mathcal{G}^{\prime}$ is $k$-tame.

Corollary 20. Let $G_{1}, \ldots, G_{k}$ be graphs and let $G$ be the join of $G_{1}, \ldots, G_{k}$. Then $s(G)=\sum_{i=1}^{k} s\left(G_{i}\right)$.

Proof. From Theorem 17 we have that $S \in \mathcal{S}_{G}$ if and only if there exists some $i \in$ $\{1, \ldots, k\}$ and some $S_{i} \in \mathcal{S}_{G_{i}}$ such that $S=S_{i} \cup\left(V(G) \backslash V\left(G_{i}\right)\right)$. Clearly, if $i, j \in$ $\{1, \ldots, k\}, i \neq j$, then $S_{i} \cup\left(V(G) \backslash V\left(G_{i}\right)\right) \neq S_{j} \cup\left(V(G) \backslash V\left(G_{j}\right)\right)$ and the sets $\left\{S_{i} \cup\right.$ $\left.\left(V(G) \backslash V\left(G_{i}\right)\right) \mid S_{i} \in \mathcal{S}_{G_{i}}\right\}$ and $\left\{S_{j} \cup\left(V(G) \backslash V\left(G_{j}\right)\right) \mid S_{j} \in \mathcal{S}_{G_{j}}\right\}$ are disjoint. Moreover, if for some $i \in\{1, \ldots, k\}$ sets $S_{i}$ and $S_{i}^{\prime}$ are distinct minimal separators in $G_{i}$, then also the sets $S_{i} \cup\left(V(G) \backslash V\left(G_{i}\right)\right)$ and $S_{i}^{\prime} \cup\left(V(G) \backslash V\left(G_{i}\right)\right)$ are distinct. Therefore $\mid\left\{S_{i} \cup(V(G) \backslash\right.$ $\left.\left.V\left(G_{i}\right)\right) \mid S_{i} \in \mathcal{S}_{G_{i}}\right\} \mid=s\left(G_{i}\right)$ for all $i \in\{1, \ldots, k\}$. It follows that $s(G)=\sum_{i=1}^{k} s\left(G_{i}\right)$, as claimed.

Corollary 20 implies the following result for graphs with disconnected complements. 
Corollary 21. Let $G$ be a graph whose complement is disconnected, with anticomponents $G_{1}, \ldots, G_{k}$. Then $s(G)=\sum_{i=1}^{k} s\left(G_{i}\right)$.

Corollary 22. Let $\mathcal{G}$ be a hereditary graph class and let $\mathcal{G}^{*}$ be the class of anticonnected graphs in $\mathcal{G}$. Then $\mathcal{G}$ is tame if and only if $\mathcal{G}^{*}$ is tame.

Proof. Since $\mathcal{G}$ is hereditary, we have $\mathcal{G}^{*} \subseteq \mathcal{G}$. Thus, if $\mathcal{G}$ is tame, then so is $\mathcal{G}^{*}$ by Observation 14. Suppose that $\mathcal{G}^{*}$ is tame. By Lemma 16, there exists a positive integer $k$ such that $s(G) \leqslant|V(G)|^{k}-1$ for all $G \in \mathcal{G}^{*}$. Let $G \in \mathcal{G} \backslash \mathcal{G}^{*}$ and let $G_{1}, \ldots, G_{p}$ (with $p \geqslant 2$ ) be the anticomponents of $G$. Since for all $i \in\{1, \ldots, p\}$ we have $G_{i} \in \mathcal{G}^{*}$, we infer using Corollary 21 and the assumption on $\mathcal{G}^{*}$ that $s(G)=\sum_{i=1}^{p} s\left(G_{i}\right) \leqslant \sum_{i=1}^{p}\left(\left|V\left(G_{i}\right)\right|^{k}-1\right) \leqslant$ $\sum_{i=1}^{p}\left|V\left(G_{i}\right)\right|^{k}-1 \leqslant\left(\sum_{i=1}^{p}\left|V\left(G_{i}\right)\right|\right)^{k}-1=|V(G)|^{k}-1$. It follows that $\mathcal{G}$ is tame.

Note that the above proof also shows that for every positive integer $k$, the class $\mathcal{G}$ is $k$-tame if and only if $\mathcal{G}^{*}$ is $k$-tame.

Next, we consider clique cutsets. A cut partition of a graph $G$ is a triple $(A, B, C)$ of pairwise disjoint subsets of $V(G)$ such that $A \cup B \cup C=V(G)$, and sets $A$ and $B$ are non-empty and anticomplete to each other. If $(A, B, C)$ is a cut partition of a graph $G$ such that $C$ is a (possibly empty) clique, we say that $C$ is a clique cutset in $G$. Recall that a graph is said to be an atom if it has no clique cutset and that, given a class $\mathcal{G}$ of graphs, we denote by $A(\mathcal{G})$ the class of all atoms that are induced subgraphs of a graph in $\mathcal{G}$.

Theorem 2 (restated). For every graph class $\mathcal{G}$, if $A(\mathcal{G})$ is tame, then so is $\mathcal{G}$.

We prove Theorem 2 using two auxiliary lemmas. The first one gives an upper bound on the number of minimal separators of a graph with a clique cutset in terms of the number of minimal separators of two smaller graphs. In the proof we use the following notation: given a graph $G$, a set $S \subseteq V(G)$, and a vertex $x \in V(G) \backslash S$, we denote by $\Gamma_{G, S, x}$ the component of $G-S$ containing $x$.

Lemma 23. Let $G$ be a graph that admits a cut partition $(A, B, C)$ such that $C$ is a clique. Then $s(G) \leqslant s(G[A \cup C])+s(G[B \cup C])+1$.

Proof. Denote by $G_{1}$ (resp. $G_{2}$ ) the subgraph of $G$ induced by $A \cup C$ (resp. $B \cup C$ ). We show the claimed inequality by proving that $\mathcal{S}_{G} \subseteq \mathcal{S}_{G_{1}} \cup \mathcal{S}_{G_{2}} \cup\{C\}$. Suppose $S$ is a minimal $x, y$-separator in $G$ and $S \neq C$. The fact that $C$ is a clique implies that either $C \cap V\left(\Gamma_{G, S, x}\right)=\emptyset$ or $C \cap V\left(\Gamma_{G, S, y}\right)=\emptyset$. By symmetry, we may assume that $C \cap V\left(\Gamma_{G, S, x}\right)=\emptyset$ and furthermore that $V\left(\Gamma_{G, S, x}\right) \subseteq A$. Since $A$ is anticomplete to $B$, this implies that $S \cap B=\emptyset$ and thus $S \subseteq V\left(G_{1}\right)$. We complete the proof by showing that $S$ is a minimal separator in $G_{1}$.

Suppose first that $S \subseteq C$. Since $S \neq C$, there exists a vertex $z \in C \backslash S$. We claim that $S$ is a minimal $x, z$-separator in $G_{1}$. Since $V\left(\Gamma_{G, S, x}\right) \subseteq A$, vertices $x$ and $z$ are separated in $G_{1}-S$. From the minimality of $S$ it follows that $\Gamma_{G, S, x}$ is an $S$-full component of $G_{1}-S$. Furthermore, since $S \subseteq C$ and $C$ is a clique in $G_{1}$, every vertex in $S$ is adjacent to $z$ in $G_{1}$. It follows that $S$ is a minimal $x, z$-separator in $G_{1}$, as claimed. 
We may thus assume that $S \nsubseteq C$ and hence $S$ contains a vertex from $A$. Since $A$ is anticomplete to $B$ and $\Gamma_{G, S, y}$ is an $S$-full component of $G-S$, this component cannot be entirely contained in $B$. Let $z$ be a vertex in $V\left(\Gamma_{G, S, y}\right) \cap V\left(G_{1}\right)$. We claim that $S$ is a minimal $x, z$-separator in $G_{1}$. Since $G_{1}$ is an induced subgraph of $G$ and $S$ separates $x$ from $z$ in $G$, we infer that $S$ is an $x, z$-separator in $G_{1}$. Note that the components of $G_{1}-S$ containing $x$ and $z$, respectively, are $\Gamma_{G, S, x}$ and the subgraph of $G_{1}$ induced by $V\left(\Gamma_{G, S, y}\right) \cap V\left(G_{1}\right)$. Thus, every vertex in $S$ is adjacent in $G_{1}$ to a vertex in $\Gamma_{G_{1}, S, x}$. It remains to show that every vertex in $S$ is adjacent in $G_{1}$ to a vertex in $\Gamma_{G_{1}, S, z}$. If $V\left(\Gamma_{G, S, y}\right) \subseteq V\left(G_{1}\right)$, then $\Gamma_{G_{1}, S, z}=\Gamma_{G, S, y}$ and the conclusion is clear. Otherwise, $V\left(\Gamma_{G, S, y}\right) \cap B \neq \emptyset$, which implies that $V\left(\Gamma_{G, S, y}\right) \cap C \neq \emptyset$. Since $S \subseteq A \cup C$ and $A$ is anticomplete to $B$, every vertex of $S$ that is adjacent in $G$ to a vertex in $V\left(\Gamma_{G, S, y}\right) \cap B$ belongs to $C$ and is therefore adjacent in $G_{1}$ also to a vertex in $V\left(\Gamma_{G, S, y}\right) \cap C \subseteq V\left(\Gamma_{G_{1}, S, z}\right)$. Therefore, using the fact that every vertex in $S$ is adjacent in $G$ to a vertex in $\Gamma_{G, S, y}$ we infer that every vertex in $S$ is adjacent in $G_{1}$ to a vertex in $\Gamma_{G_{1}, S, z}$, as claimed.

Recall that a graph class $\mathcal{G}$ is said to be $k$-tame if $s(G) \leqslant|V(G)|^{k}-1$ for every graph $G \in \mathcal{G}$ and that, by Lemma 16, a graph class is tame if and only if it is $k$-tame for some $k \in \mathbb{N}$. Thus, Theorem 2 is a direct consequence of Lemma 16 the following lemma.

Lemma 24. Let $\mathcal{G}$ be a graph class such that the class of atoms $A(\mathcal{G})$ is $k$-tame for some non-negative integer $k$. Then $\mathcal{G}$ is $(k+1)$-tame.

Proof. Let $\mathcal{G}$ and $k$ be as in the lemma. We show that every $n$-vertex graph $G \in \mathcal{G}$ has at most $n^{k+1}-1$ minimal separators. The proof is by induction on $n$.

If $n=1$, then $G=K_{1}$ and $G$ has $0=1^{k+1}-1$ minimal separators. Suppose that $n>1$ and let $G$ be an $n$-vertex graph from $\mathcal{G}$. If $G$ is an atom, then $G$ has at most $n^{k}-1$ minimal separators by assumption, and $n^{k}-1<n^{k+1}-1$. Suppose now that $G$ has a clique cutset. Then, $G$ has a cut partition $(A, B, C)$ such that $C$ is a clique and $G_{A}=G[A \cup C]$ has no clique cutset (see, e.g., $[10,51]$ ). Since $G_{A}$ belongs to $A(\mathcal{G})$, we have $s\left(G_{A}\right) \leqslant\left|V\left(G_{A}\right)\right|^{k}-1$. Furthermore, since $B$ is non-empty, we have $\left|V\left(G_{A}\right)\right| \leqslant n-1$ and consequently, $s\left(G_{A}\right) \leqslant(n-1)^{k}-1$. Note that also $A$ is non-empty, and hence we can apply the induction hypothesis to the graph $G_{B}=G[B \cup C] \in \mathcal{G}$ to derive $s\left(G_{B}\right) \leqslant\left|V\left(G_{B}\right)\right|^{k+1}-1 \leqslant(n-1)^{k+1}-1$. By Lemma 23 , we have

$$
s(G) \leqslant s\left(G_{A}\right)+s\left(G_{B}\right)+1 \leqslant(n-1)^{k}+(n-1)^{k+1}-1 .
$$

Thus, to complete the proof, it suffices to show the following inequality:

$$
n^{k+1}-(n-1)^{k+1} \geqslant(n-1)^{k} .
$$

Note that for every two non-negative real numbers $a$ and $b$ we have

$$
a^{k+1}-b^{k+1}=(a-b) \cdot\left(\sum_{i=0}^{k} a^{k-i} b^{i}\right) \geqslant(a-b) b^{k} .
$$

Applying the inequality $a^{k+1}-b^{k+1} \geqslant(a-b) b^{k}$ to $a=n, b=n-1$ establishes (1). 


\section{Sufficient conditions}

We identify several sufficient conditions for a graph class to be tame. The first two reveal two infinite families of tame graph classes, each parameterized by two positive integers.

\subsection{Graphs in which all edges are almost dominating}

Our first sufficient condition is an easy consequence of Ramsey's theorem.

Theorem 5 (restated). For every two positive integers $k$ and $\ell$, the class of $\left\{P_{2}+k P_{1}, K_{\ell}+\right.$ $P_{2}$ \}-free graphs is tame.

We prove the theorem using an auxiliary lemma. For a non-negative integer $k$, we denote by $\mathcal{C}_{k}$ the class of graphs $G$ such that for every edge $u v \in E(G)$, at most $k$ vertices of $G$ are adjacent to neither $u$ nor $v$.

Lemma 25. For every positive integer $k$, the class $\mathcal{C}_{k}$ is tame.

Proof. Since $\mathcal{C}_{i} \subseteq \mathcal{C}_{i+1}$ for all $i \geqslant 0$, we may assume that $k \geqslant 1$. We will prove that for every minimal separator $S$ in $G$, there exists a set $X \subseteq V(G)$ such that $|X| \leqslant k$ and $S=N_{G}(X)$. Clearly, this will imply that $G$ has at most $\left(\begin{array}{c}|V(G)| \\ k\end{array}\right)$ minimal separators. Let $S$ be a minimal separator in $G$ and let $C$ and $D$ be two $S$-full components of $G-S$. Since $N_{G}(V(C))=N_{G}(V(D))=S$, it suffices to show that $|V(C)| \leqslant k$ or $|V(D)| \leqslant k$. Suppose that this is not the case. Then $|V(C)| \geqslant k+1$ and $|V(D)| \geqslant k+1$. Since $|V(C)| \geqslant k+1 \geqslant 2$ and $C$ is connected, there is an edge $u v \in E(C)$. But then, $G$ contains at least $|V(D)| \geqslant k+1$ vertices that are adjacent to neither $u$ nor $v$, contrary to the fact that $G \in \mathcal{C}_{k}$.

Proof of Theorem 5. Let $G$ be a $\left\{P_{2}+k P_{1}, K_{\ell}+P_{2}\right\}$-free graph and let $r=R(\ell, k)$. By Lemma 25, it suffices to show that $G \in \mathcal{C}_{r-1}$. Suppose this is not the case. Then, $G$ has an edge $u v$ such that there exists a set $X$ of $r$ vertices of $G$ such that every vertex in $X$ is adjacent to neither $u$ nor $v$. By Ramsey's theorem, there exists a set $Z \subseteq X$ such that $Z$ is either a clique of size $\ell$ or an independent set of size $k$ in $G$. But then the set $\{u, v\} \cup Z$ induces either a $K_{\ell}+P_{2}$ or $P_{2}+k P_{1}$, respectively. Both cases lead to a contradiction.

\subsection{Graphs of bounded clique cover number excluding some complete prism}

We now prove Theorem 3. We reformulate it in an equivalent way that will facilitate our inductive proof. We denote by $L_{k}$ the complete prism of order $k$. For every two positive integers $k$ and $\ell$, let $\mathcal{C}_{k, \ell}$ denote the class of all $L_{k}$-free graphs with clique cover number at most $\ell$. Theorem 3 is equivalent to the following.

Theorem 26. For every two positive integers $k$ and $\ell$, the class $\mathcal{C}_{k, \ell}$ is tame.

We prove Theorem 26 by induction on $\ell$, with cases $\ell \in\{1,2\}$ as the base cases. In the proof for the case $\ell=2$, we make use of the following result, discovered independently by Alekseev [1], Balas-Yu [3], and Prisner [44]. 
Theorem 27. For every positive integer $k$, every $\overline{k P_{2}}$-free graph $G$ has $O\left(|V(G)|^{2 k-2}\right)$ maximal cliques.

Lemma 28. For every positive integer $k$, the class $\mathcal{C}_{k, 2}$ is tame.

Proof. Let $G$ be an $L_{k}$-free graph with clique cover number at most 2 and let $\left\{A_{1}, A_{2}\right\}$ be a clique cover of $G$. We associate to $G$ a graph $G^{\prime}$ obtained by swapping the roles of edges and non-edges between cliques $A_{1}$ and $A_{2}$. Formally, $G^{\prime}$ is defined as follows: $V\left(G^{\prime}\right)=V(G)$ and $E\left(G^{\prime}\right)=E_{1} \cup E_{2} \cup E_{3}$ where $E_{1}=\left\{u v \mid u, v \in A_{1}, u \neq v\right\}, E_{2}=\{u v \mid$ $\left.u, v \in A_{2}, u \neq v\right\}$, and $E_{3}=\left\{u v \mid u \in A_{1}, v \in A_{2}, u v \notin E(G)\right\}$.

We prove that $G$ has a polynomially bounded number of minimal separators in two steps. First we prove that if a set $S \subseteq V(G)$ is a minimal separator in $G$, then its complement $\bar{S}=V(G) \backslash S$ is a maximal clique in $G^{\prime}$. Then, we show that $G^{\prime}$ is $\overline{k P_{2}}$-free. Finally, we invoke the result of Theorem 27 to infer that $G^{\prime}$ has $O\left(\left|V\left(G^{\prime}\right)\right|^{2 k-2}\right)=$ $O\left(|V(G)|^{2 k-2}\right)$ maximal cliques. Since the mapping $S \mapsto \bar{S}$ is one-to-one, this will imply that $G$ has $O\left(|V(G)|^{2 k-2}\right)$ minimal separators.

Let $S$ be a minimal separator in $G$. Then $G-S$ has precisely two components, namely $G\left[A_{1} \backslash S\right]$ and $G\left[A_{2} \backslash S\right]$, and both of these components are $S$-full. Since sets $A_{1} \backslash S$ and $A_{2} \backslash S$ are anticomplete to each other in $G$, we infer that $\bar{S}=\left(A_{1} \backslash S\right) \cup\left(A_{2} \backslash S\right)$ is a clique in $G^{\prime}$. It remains to prove that $\bar{S}$ is a maximal clique. Assume for a contradiction that there exists a vertex $x \in S$ such that $\bar{S} \cup\{x\}$ is a clique in $G^{\prime}$. By symmetry we may assume that $x \in A_{1} \cap S$. Since $A_{2} \backslash S$ is an $S$-full component in $G-S$, vertex $x$ is adjacent in $G$ to some vertex $y \in A_{2} \backslash S$. This implies that vertices $x$ and $y$ are non-adjacent in $G^{\prime}$, contradicting the fact that they both belong to clique $\bar{S} \cup\{x\}$. It follows that $\bar{S}$ is a maximal clique in $G^{\prime}$, as claimed.

To complete the proof of the lemma, it remains to show that $G^{\prime}$ is $\overline{k P_{2}}$-free. Assume the opposite: let $X \subseteq A_{1}, Y \subseteq A_{2}$ be such that $G^{\prime}[X \cup Y] \cong \overline{k P_{2}}$. Since $X$ and $Y$ are cliques in $G^{\prime}$, all the non-edges of the $\overline{k P_{2}}$ must go from $X$ to $Y$. It follows that $G[X \cup Y] \cong L_{k}$, contradicting the fact that $G$ is $L_{k}$-free. Hence, $G^{\prime}$ is $\overline{k P_{2}}$-free, as claimed.

Before proceeding to the induction step, we prove two more technical results. For two positive integers $k, \ell$, let us denote by $\mathcal{C}_{k, \ell}^{*}$ the class of all graphs of the form $G-S^{*}$ such that $G \in \mathcal{C}_{k, \ell}$ and there exists a clique cover $\left\{A_{1}, \ldots, A_{\ell}\right\}$ of $G$ such that $S^{*}$ is a minimal separator in the graph $G-A_{\ell}$.

Lemma 29. Suppose that for some positive integers $k, \ell$ with $\ell \geqslant 3$, the classes $\mathcal{C}_{k, \ell-1}$ and $\mathcal{C}_{k, \ell}^{*}$ are both tame. Then, the class $\mathcal{C}_{k, \ell}$ is also tame.

Proof. Fix positive integers $k, \ell$ with $\ell \geqslant 3$ and suppose that the classes $\mathcal{C}_{k, \ell-1}$ and $\mathcal{C}_{k, \ell}^{*}$ are $a$-tame and $b$-tame, respectively. We want to prove that $\mathcal{C}_{k, \ell}$ is tame as well. Let $G$ be a graph in $\mathcal{C}_{k, \ell}$ and let $\left\{A_{1}, A_{2}, \ldots, A_{\ell}\right\}$ be a clique cover of $G$. Let $S$ be a minimal separator in $G$ and let $C$ and $D$ be two distinct $S$-full components in $G-S$. Since $\ell \geqslant 3$, there exists at least one clique $A_{i}, i \in\{1, \ldots, \ell\}$, such that $V(C) \nsubseteq A_{i}$ and $V(D) \nsubseteq A_{i}$. By renumbering the cliques if necessary, we may assume that $V(C) \nsubseteq A_{\ell}$ and $V(D) \nsubseteq A_{\ell}$. Furthermore, since $C$ and $D$ are anticomplete to each other and $A_{\ell}$ is a clique, we have $V(C) \cap A_{\ell}=\emptyset$ or $V(D) \cap A_{\ell}=\emptyset$. By symmetry, we may assume that $V(C) \cap A_{\ell}=\emptyset$. 
Consider the graph $G-A_{\ell}$. Fix two vertices $u \in V(C)$ and $v \in V(D) \backslash A_{\ell}$. From the definition of $S$ it follows that $S \backslash A_{\ell}$ is a $u, v$-separator in $G-A_{\ell}$. Let $S^{*} \subseteq S \backslash A_{\ell}$ be a minimal $u, v$-separator in $G-A_{\ell}$. By Corollary 11 it follows that the set $S^{\prime}:=S \backslash S^{*}$ is a minimal separator in $G-S^{*}$. In particular, we have $S=S^{*} \cup S^{\prime}$ where $S^{*} \in \mathcal{S}_{G-A_{\ell}}$ and $S^{\prime} \in \mathcal{S}_{G-S^{*}}$. Clearly, $G-A_{\ell} \in \mathcal{C}_{k, \ell-1}$. It follows that every minimal separator $S$ in $G$ can be written as a union of two sets $S^{*}$ and $S^{\prime}$ such that $S^{*} \in \mathcal{S}_{G^{*}}$ where $G^{*}=G-A_{\ell} \in \mathcal{C}_{k, \ell-1}$ and $S^{\prime} \in \mathcal{S}_{G^{\prime}}$ where $G^{\prime}=G-S^{*} \in \mathcal{C}_{k, \ell}^{*}$.

Note that the above arguments hold for any clique cover $\left\{A_{1}, A_{2}, \ldots, A_{\ell}\right\}$ of $G$, except that some renaming of cliques might have been necessary depending on $S, C$, and $D$, to assure that $V(C) \cap A_{\ell}=\emptyset$ and $V(D) \nsubseteq A_{\ell}$. Once a clique cover $\left\{A_{1}, A_{2}, \ldots, A_{\ell}\right\}$ of $G$ is fixed, there are $\ell$ choices for which clique in the cover is labeled $A_{\ell}$. Furthermore, since $\mathcal{C}_{k, \ell-1}$ is $a$-tame, there are at most $\left|V\left(G^{*}\right)\right|^{a}-1 \leqslant|V(G)|^{a}-1$ choices for $S^{*}$. The graph $G^{\prime} \in \mathcal{C}_{k, \ell}^{*}$ as above is uniquely determined with $G$ and $S^{*}$ and since $\mathcal{C}_{k, \ell}^{*}$ is $b$-tame, there are at most $\left|V\left(G^{\prime}\right)\right|^{b}-1 \leqslant|V(G)|^{b}-1$ choices for $S^{\prime}$. Since $S=S^{*} \cup S^{\prime}$, we infer that altogether we have at most $\ell \cdot\left(|V(G)|^{a}-1\right)\left(|V(G)|^{b}-1\right)$ choices for $S$. This shows that $s(G) \leqslant \ell\left(|V(G)|^{a}-1\right)\left(|V(G)|^{b}-1\right)$ and thus $\mathcal{C}_{k, \ell}$ is tame.

Lemma 30. For every two positive integers $k$ and $\ell$ with $\ell \geqslant 3$, if the class $\mathcal{C}_{k, \ell-1}$ is tame, then so is the class $\mathcal{C}_{k, \ell}^{*}$.

Proof. Fix positive integers $k$ and $\ell$ with $\ell \geqslant 3$ and suppose that the class $\mathcal{C}_{k, \ell-1}$ is tame. By Theorem 2, to show that $\mathcal{C}_{k, \ell}^{*}$ is tame, it suffices to show that the class $A\left(\mathcal{C}_{k, \ell}^{*}\right)$ is tame. We do so by showing that every graph in $A\left(\mathcal{C}_{k, \ell}^{*}\right)$ belongs to $\mathcal{C}_{k, \ell-1}$. This will suffice since the class $\mathcal{C}_{k, \ell-1}$ is tame by assumption.

Let $G \in A\left(\mathcal{C}_{k, \ell}^{*}\right)$. Then $G$ is an atom that is an induced subgraph of a graph $G^{*} \in \mathcal{C}_{k, \ell}^{*}$. By definition of $\mathcal{C}_{k, \ell}^{*}$, there exist a graph $G^{\prime}$ in $\mathcal{C}_{k, \ell}$, a clique cover $\left\{A_{1}, \ldots, A_{\ell}\right\}$ of $G^{\prime}$, and a minimal separator $S^{*}$ in the graph $G^{\prime}-A_{\ell}$ such that $G^{*}=G^{\prime}-S^{*}$. The fact that $S^{*}$ is a separator in $G^{\prime}-A_{\ell}$ implies that the graph $G^{\prime}-S^{*}-A_{\ell}=G^{*}-A_{\ell}$ is disconnected. Thus, there exists a cut partition $(A, B, C)$ of $G^{*}$ such that $C=A_{\ell}$. Note that sets $A \cap V(G)$ and $B \cap V(G)$ are anticomplete to each other in $G$. Note also that $A_{\ell} \cap V(G)$ is a (possibly empty) clique in $G$. Since $G$ has no clique cutset, we infer that one of the sets $A \cap V(G)$ and $B \cap V(G)$ is empty, say $A \cap V(G)=\emptyset$. Furthermore, since the sets $A$ and $B$ are anticomplete to each other in $G^{*}-A_{\ell}$, it follows that $A$ is the union of a nonempty subset of the set of cliques $\left\{A_{1} \backslash S^{*}, \ldots, A_{\ell-1} \backslash S^{*}\right\}$. Let $i \in\{1, \ldots, \ell-1\}$ be such that $A_{i} \backslash S^{*} \subseteq A$. The fact that $A \cap V(G)=\emptyset$ now implies that the vertex set of $G$ can be covered with $\ell-1$ cliques $\left(A_{j} \backslash S^{*}\right) \cap V(G)$, for $j \in\{1, \ldots, \ell\} \backslash\{i\}$. Since $G$ is an induced subgraph of a graph in $\mathcal{C}_{k, \ell}$, it is $L_{k}$-free. Consequently, $G \in \mathcal{C}_{k, \ell-1}$ and the proof is complete.

Proof of Theorem 26. Fix a positive integer $k$. We prove that for every positive integer $\ell$, the class $\mathcal{C}_{k, \ell}$ is tame, using induction on $\ell$. If $\ell=1$, then every graph in $\mathcal{C}_{k, \ell}$ is complete and $\mathcal{C}_{k, \ell}$ is tame. If $\ell=2$, then the class $\mathcal{C}_{k, \ell}$ is tame by Lemma 28. Suppose now that $\ell \geqslant 3$ and that the class $\mathcal{C}_{k, \ell-1}$ is tame. By Lemma 30, the class $\mathcal{C}_{k, \ell}^{*}$ is tame. Since the classes $\mathcal{C}_{k, \ell-1}$ and $\mathcal{C}_{k, \ell}^{*}$ are tame, Lemma 29 implies that so is the class $\mathcal{C}_{k, \ell}$. 


\subsection{Subclasses of $C_{4}$-free graphs}

We now derive two consequences of Theorem 26 dealing with subclasses of $C_{4}$-free graphs. Wagon proved in [53] that every $2 P_{2}$-free graph with clique number $k$ is $(k(k+1) / 2)$-colorable. This result can be equivalently stated as follows.

Theorem 31. For every positive integer $k$, every $\left\{k P_{1}, C_{4}\right\}$-free graph has clique cover number at most $k(k-1) / 2$.

Since the graph $L_{2}$ is the 4 -cycle, this result implies that the class of $\left\{k P_{1}, C_{4}\right\}$-free graphs is a subclass of the class $\mathcal{C}_{2, k(k-1) / 2}$. By Theorem 26, the class $\mathcal{C}_{2, k(k-1) / 2}$ is tame, and we thus obtain the following.

Corollary 32. For every positive integer $k$, the class of $\left\{k P_{1}, C_{4}\right\}$-free graphs is tame.

The question of which classes of graphs defined by a set of forbidden induced subgraphs with at most four vertices are tame was first investigated in the early version of this paper [37]. An almost complete dichotomy was obtained, leaving open only two cases: the classes of $\left\{4 P_{1}, C_{4}\right\}$-free and $\left\{4 P_{1}\right.$, claw, $\left.C_{4}\right\}$-free graphs. Clearly, the result of Corollary 32 resolves both cases. ${ }^{1}$ We now generalize Corollary 32 by replacing $k P_{1}$ in the statement of the corollary with $P_{2}+k P_{1}$.

Theorem 4 (restated). For every positive integer $k$, the class of $\left\{P_{2}+k P_{1}, C_{4}\right\}$-free graphs is tame.

Proof. Let $G$ be a $\left\{P_{2}+k P_{1}, C_{4}\right\}$-free graph. Fix a maximum independent set $I$ in $G$. If $|I| \leqslant 2 k-1$, then $G$ is $\left\{2 k P_{1}, C_{4}\right\}$-free and Corollary 32 implies that $G$ has a polynomially bounded number of minimal separators. Thus, in what follows we assume that $|I| \geqslant 2 k$. Let $w$ be a vertex in $V(G) \backslash I$. Since $I$ is a maximal independent set, $w$ has a neighbor in $I$. Consequently, since $G$ is $\left(P_{2}+k P_{1}\right)$-free, $w$ has at most $k-1$ non-neighbors in $I$ and hence $w$ has at least $|I|-(k-1) \geqslant k+1$ neighbors in $I$. Suppose next that $u$ and $v$ are two distinct non-adjacent vertices in $V(G) \backslash I$. Since each of $u$ and $v$ has at least $k+1$ neighbors in $I$, they have at least two common neighbors in $I$. But this contradicts the fact that $G$ is $C_{4}$-free. It follows that $V(G) \backslash I$ is a clique. Since $I$ is an independent set and $V(G) \backslash I$ is a clique, $G$ is $2 P_{2}$-free, and we infer from Lemma 12 that $G$ has at most $|V(G)|$ minimal separators.

\section{$5 \quad$ Necessary conditions}

We now turn to necessary conditions for a graph class to be tame. We give two structurally different constructions of graphs with exponentially many minimal separators. The first construction involves families of graphs of arbitrarily large maximum degree but without long induced paths. The second construction involves two families of graphs with small

\footnotetext{
${ }^{1}$ Daniel Lokshtanov kindly communicated to us that the result of Corollary 32 was also obtained independently (and at approximately the same time) by Peter Gartland.
} 
maximum degree but with arbitrarily long induced paths. In both cases, we make use of line graphs.

Given positive integers $k$ and $\ell$, the $k, \ell$-theta graph is the graph $\theta_{k, \ell}$ obtained as the union of $k$ internally disjoint paths of length $\ell$ with common endpoints $a$ and $b$. For every positive integer $\ell$, we define a family of graphs $\Theta_{\ell}$ in the following way: $\Theta_{\ell}=\left\{\theta_{k, \ell} \mid k \geqslant 2\right\}$. Note that $\ell$ refers to the length of each of the $a, b$-paths and not to the number of paths, which is unrestricted.

Observation 33. For every integer $\ell \geqslant 3$, the class $\Theta_{\ell}$ is not tame.

Proof. Let $k \geqslant 2, \ell \geqslant 3$, let $G=\theta_{k, \ell}$, and let $P^{1}, \ldots, P^{k}$ be paths in $G$ as in the definition of the theta graphs. Let $S$ be any set of vertices of $G$ containing exactly one internal vertex of each of the paths $P^{j}$. Then, the graph $G-S$ has two $S$-full components and Lemma 9 implies that $S$ is a minimal separator in $G$. Note that for every $j \in\{1, \ldots, k\}$, path $P^{j}$ has exactly $\ell-1$ internal vertices. It follows that $s\left(\theta_{k, \ell}\right) \geqslant(\ell-1)^{k}$. Thus, as $\left|V\left(\theta_{k, \ell}\right)\right|=k(\ell-1)+2$, we infer that for every fixed positive integer $\ell \geqslant 3$, the class $\Theta_{\ell}$ is not tame.

Corollary 34. If $\mathcal{G}$ is graph class such that $\Theta_{\ell} \subseteq \mathcal{G}$ for some $\ell \geqslant 3$, then $\mathcal{G}$ is not tame.

Consider now the family of line graphs of theta graphs. More precisely, given positive integers $k$ and $\ell$, let $L_{k, \ell}$ denote the line graph of $\theta_{k, \ell}$ and let $\mathcal{L}_{\ell}=\left\{L_{k, \ell} \mid k \geqslant 2\right\}$. Note that the class $\mathcal{L}_{2}$ is precisely the class of all complete prisms of order at least two.

Observation 35. For every integer $\ell \geqslant 2$, the class $\mathcal{L}_{\ell}$ is not tame.

Proof. Let $k, \ell \geqslant 2$ and let $G=L_{k, \ell}$. Then, graph $G$ consists of two cliques $K$ and $K^{\prime}$, each of size $k$, say with $K=\left\{a_{1}, \ldots, a_{k}\right\}$ and $K^{\prime}=\left\{b_{1}, \ldots, b_{k}\right\}$, and $k$ internally pairwise disjoint paths $P^{1}, \ldots, P^{k}$ such that for every $j \in\{1, \ldots, k\}$, path $P^{j}$ is an $a_{i}, b_{i}$-path with $\left|V\left(P^{j}\right)\right|=\ell, V\left(P^{j}\right) \cap K=\left\{a_{j}\right\}$ and $V\left(P^{j}\right) \cap K^{\prime}=\left\{b_{j}\right\}$. Consider any set $S$ of vertices of $G$ containing exactly one vertex from each of the paths $P^{j}$ and such that $S \notin\left\{K, K^{\prime}\right\}$. Then, the graph $G-S$ has two $S$-full components and Lemma 9 implies that $S$ is a minimal separator in $G$. It follows that $s\left(L_{k, \ell}\right) \geqslant \ell^{k}-2$. Thus, as $\left|V\left(L_{k, \ell}\right)\right|=k(\ell+1)$, we infer that for every fixed positive integer $\ell \geqslant 2$, the class $\mathcal{L}_{\ell}$ is not tame.

Corollary 36. If $\mathcal{G}$ is a graph class such that $\mathcal{L}_{\ell} \subseteq \mathcal{G}$ for some $\ell \geqslant 2$, then $\mathcal{G}$ is not tame.

Corollary 37. The class of $\left\{3 P_{1}\right.$, diamond $\}$-free graphs is not tame.

Proof. Let $G \in \mathcal{L}_{2}$. Since the vertex set of $G$ is the union of two cliques, $G$ is $3 P_{1}$-free. Moreover, it is not difficult to see that $G$ is diamond-free. Consequently, the class of $\left\{3 P_{1}\right.$, diamond -free graphs contains all graphs in $\mathcal{L}_{2}$ and is therefore not tame by Corollary 36 .

Corollaries 34 and 36 also imply the following general necessary condition for a finite set $\mathcal{F}$ of graphs such that the class of $\mathcal{F}$-free graphs is tame. 
Proposition 38. Let $\mathcal{F}$ be a finite set of graphs such that the class of $\mathcal{F}$-free graphs is tame. Then $\mathcal{F}$ either contains an induced subgraph of $P_{4}$ or of $2 P_{2}$, or $\mathcal{F}$ contains both an acyclic graph and a graph of girth at most 4 .

Proof. Let $\mathcal{F}$ be a finite set of graphs such that for every $F \in \mathcal{F}$ we have $F \nsubseteq_{i} P_{4}$, $F \nsubseteq_{i} 2 P_{2}$. Suppose for a contradiction that either all graphs in $\mathcal{F}$ contain cycles or all of them are of girth more than 5 . We analyze the two cases separately.

Case 1: all graphs in $\mathcal{F}$ contain cycles. Let $\ell$ be the smallest integer such that $\ell \geqslant 3$ and for every graph $F \in \mathcal{F}$, it holds that $F$ does not contain an induced cycle of length exactly $2 \ell$. Note that $\ell$ is well defined since $\mathcal{F}$ is finite. We claim that every graph in $\Theta_{\ell}$ is $\mathcal{F}$-free. Suppose for a contradiction that for some $k \geqslant 2$, the graph $\theta_{k, \ell}$ contains an induced subgraph isomorphic to some $F \in \mathcal{F}$. Since $F$ contains an induced cycle and every induced cycle contained in $\theta_{k, \ell}$ is of length $2 \ell$, we infer that $F$ contains an induced cycle of length $2 \ell$. However, this contradicts the definition of $\ell$. Thus, every graph in $\Theta_{\ell}$ is $\mathcal{F}$-free, as claimed. By Corollary 34, the class of $\mathcal{F}$-free graphs is not tame, a contradiction.

Case 2: every graph in $\mathcal{F}$ is of girth more than five. We will show that in this case, every graph in $\mathcal{L}_{2}$ is $\mathcal{F}$-free. By Corollary 36 this will imply that the class of $\mathcal{F}$-free graphs is not tame, a contradiction. From the definition of $\mathcal{L}_{2}$ it follows that every graph in $\mathcal{L}_{2}$ has independence number two. Thus, to show that every graph in $\mathcal{L}_{2}$ is $\mathcal{F}$-free, it suffices to prove that $\alpha(F) \geqslant 3$ for all $F \in \mathcal{F}$. Suppose for a contradiction that $\alpha(F) \leqslant 2$ for some $F \in \mathcal{F}$. Then $F$ is acyclic, since otherwise a shortest cycle in $F$ would be of length at least 6 , which would imply $\alpha(F) \geqslant 3$. Moreover, $F$ has at most two connected components. If $F$ is connected, then $F$ is a tree with $\alpha(F) \leqslant 2$. In particular, the maximum degree of $F$ is at most 2 , hence $F$ a path with at most four vertices, which implies $F \subseteq_{i} P_{4}$, a contradiction. If $F$ has exactly two components, then the condition $\alpha(F) \leqslant 2$ implies that each component of $F$ is a complete graph. However, since $F$ is acyclic, each component of $F$ is induced subgraph of $P_{2}$. It follows that $F \subseteq_{i} 2 P_{2}$, a contradiction.

We now turn to the second type of construction for families of graphs with exponentially many minimal separators. Let $r, s \geqslant 2$ be integers. An $r \times s$-grid is the graph with vertex set $\{0, \ldots, r-1\} \times\{0, \ldots, s-1\}$ in which two vertices $(i, j)$ and $\left(i^{\prime}, j^{\prime}\right)$ are adjacent if and only if $\left|i-i^{\prime}\right|+\left|j-j^{\prime}\right|=1$. Given an integer $h \geqslant 2$, an elementary wall of height $h$ is the graph $W_{h}$ obtained from the $(2 h+2) \times(h+1)$-grid by deleting all edges with endpoints $(2 i+1,2 j)$ and $(2 i+1,2 j+1)$ for all $i \in\{0,1, \ldots, h\}$ and $j \in\{0,1, \ldots,\lfloor(h-1) / 2\rfloor\}$, deleting all edges with endpoints $(2 i, 2 j-1)$ and $(2 i, 2 j)$ for all $i \in\{0,1, \ldots, h\}$ and $j \in\{1, \ldots,\lfloor h / 2\rfloor\}$, and deleting the two resulting vertices of degree one. Note that an elementary wall of height $h$ consists of $h$ levels each containing $h$ bricks, where a brick is a cycle of length six; see Fig. 3(a).

Grids contain exponentially many minimal separators [49]. We show next that the same is true for walls.

Proposition 39. For every integer $h \geqslant 2$, an elementary wall of height $h$ has at least $2^{h}$ minimal separators. 


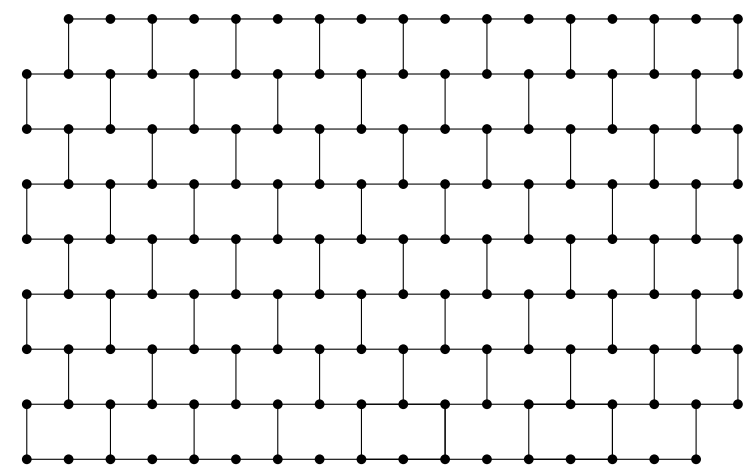

(a)

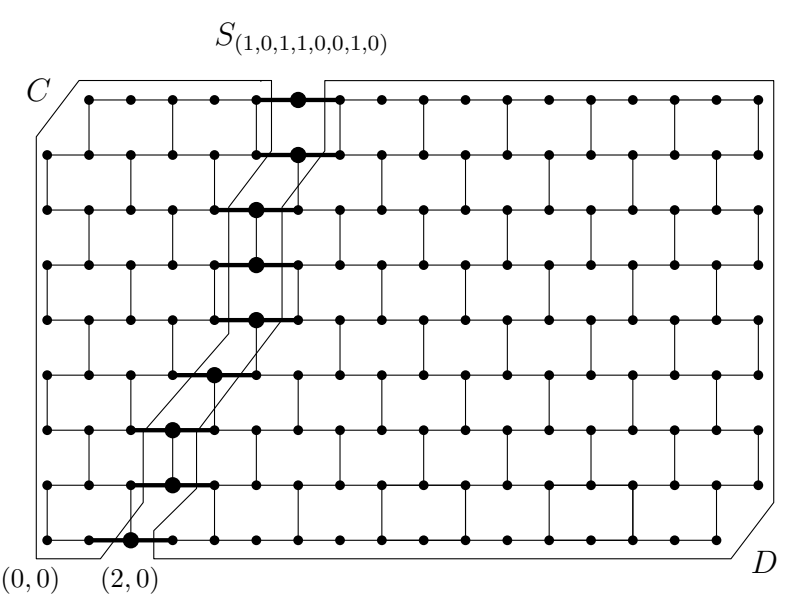

(b)

Figure 3: (a) An elementary wall of height 8. (b) A minimal separator $S_{(1,0,1,1,0,0,1,0)}$ in $W_{8}$ and the two components of $W_{8}-S_{(1,0,1,1,0,0,1,0)}$.

Proof. Fix an integer $h \geqslant 2$. We will define a family of $2^{h}$ subsets of $V\left(W_{h}\right)$ and show that each of them is a minimal separator in $W_{h}$. For each binary sequence of length $h$, say $x=$ $\left(x_{1}, \ldots, x_{h}\right) \in\{0,1\}^{h}$, we define a set $S_{x}$ by the following rule: $S_{x}=\left\{v^{x, 0}, v^{x, 1}, \ldots, v^{x, h}\right\}$ where $v^{x, 0}=(2,0)$ (independently of $x$ ) and for all $j \in\{1, \ldots, h\}$, we set $v^{x, j}=v^{x, j-1}+$ $\left(x_{j}, 1\right)$, where addition is performed component-wise. Clearly, for each $x \in\{0,1\}^{h}$ and each $j \in\{1, \ldots, h\}$, we have $v^{x, j}=\left(\sum_{i=1}^{j} x_{i}+2, j\right) \leqslant(h+2, h)$, where comparison is performed component-wise. It follows that $S_{x} \subseteq V\left(W_{h}\right)$. Moreover, the graph $W_{h}-S_{x}$ has exactly two connected components, say $C$ and $D$, with $V(C)=\bigcup_{j=0}^{h}\left\{(i, j) \in V\left(W_{h}\right) \mid\right.$ $\left.i<v_{1}^{x, j}\right\}$ and $V(D)=\bigcup_{j=0}^{h}\left\{(i, j) \in V\left(W_{h}\right) \mid i>v_{1}^{x, j}\right\}$. Note that each vertex $v^{x, j} \in S_{x}$ has a neighbor in $C$, namely $v^{x, j}-(1,0)$, and a neighbor in $D$, namely $v^{x, j}+(1,0)$. By Lemma 9 , set $S_{x}$ is a minimal separator in $W_{h}$. Since the sets $S_{x}$ are pairwise distinct, this completes the proof. Fig. 3(b) shows an example with $h=4$ and $x=(1,0,1,1)$. The thick horizontal edges can be used to justify the fact that $C$ and $D$ are $S_{x}$-full components of $W_{h}-S_{x}$.

Another family with exponentially many minimal separators is given by the line graphs of elementary walls; see Fig. 4(a) for an example.

Proposition 40. For every even integer $h \geqslant 2$, the graph $L\left(W_{h}\right)$ has at least $2^{h / 2}$ minimal separators.

Proof. We use a modification of the construction used in the proof of Proposition 39. We again consider the minimal separators $S_{x}$ in $W_{h}$ constructed in the proof of Proposition 39; however, for technical reasons that will simplify the argument, we restrict ourselves only to the $2^{h / 2}$ minimal separators $S_{x}$ in $W_{h}$ that arise from binary sequences $x \in X_{h}$, where

$$
X_{h}=\left\{\left(x_{1}, \ldots, x_{h}\right) \in\{0,1\}^{h} \mid x_{2 i-1}=x_{2 i} \text { for all } i \in\{1,2, \ldots, h / 2\}\right\} .
$$




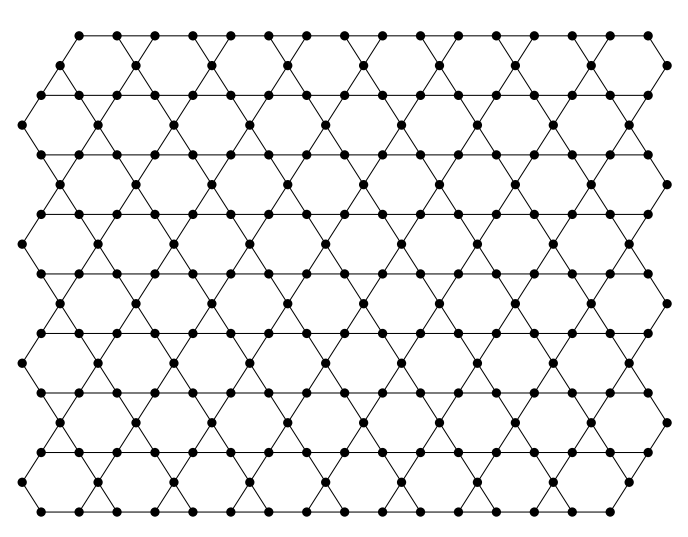

(a)

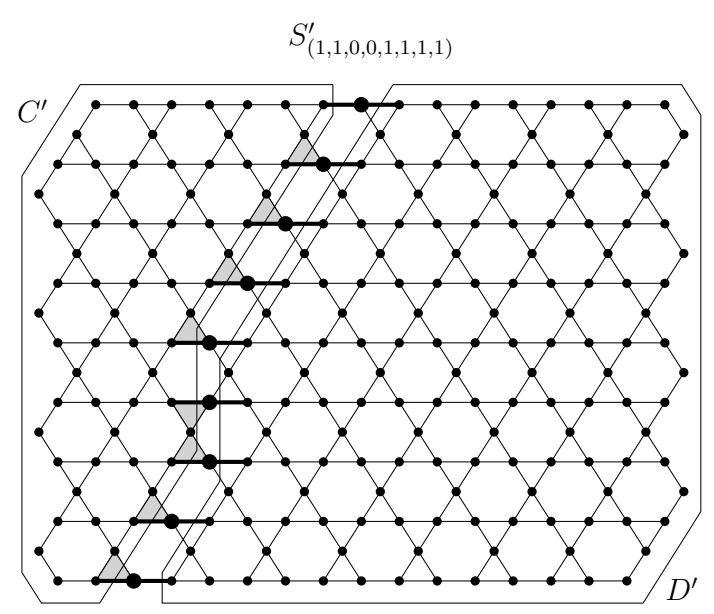

(b)

Figure 4: (a) $L\left(W_{8}\right)$, the line graph of an elementary wall of height 8 . (b) The set of nine vertices depicted with large black disks is a minimal separator $S_{(1,1,0,0,1,1,1,1)}^{\prime}$ in $L\left(W_{8}\right)$, which corresponds to the minimal separator $S_{(1,1,0,0,1,1,1,1)}$ in $W_{8}$. The two components of $L\left(W_{8}\right)-S_{(1,1,0,0,1,1,1,1)}^{\prime}$ are also depicted.

Recall that for every $x \in X_{h}$, we have $S_{x}=\left\{v^{x, 0}, v^{x, 1}, \ldots, v^{x, h}\right\}$ where $v^{x, 0}=(2,0)$ and $v^{x, j}=v^{x, j-1}+\left(x_{j}, 1\right)$ for all $j \in\{1, \ldots, h\}$. A set of $2^{h / 2}$ minimal separators of $L\left(W_{h}\right)$ can be obtained as follows. For each $x \in X_{h}$, we define a set $S_{x}^{\prime} \subseteq V\left(L\left(W_{h}\right)\right)$ as follows: $S_{x}^{\prime}=\left\{e^{x, j} \mid v^{x, j} \in S_{x}\right\}$ where $e^{x, j}$ is the vertex of the line graph of $W_{h}$ corresponding to the edge in $W_{h}$ joining vertex $v^{x, j}$ with vertex $v^{x, j}+(1,0)$.

Since the mapping is one-to-one, the set $\left\{S_{x}^{\prime} \mid x \in X_{h}\right\}$ is of cardinality $2^{h / 2}$. Therefore, to complete the proof it suffices to show that for every $x \in X_{h}$, set $S_{x}^{\prime}$ is a minimal separator in $L\left(W_{h}\right)$. Let us first argue that the graph $L\left(W_{h}\right)-S_{x}^{\prime}$ is disconnected. Vertices of the wall $W_{h}$ correspond bijectively to maximal cliques of its line graph. For every $x \in X_{h}$, every vertex of the form $v^{x, j}$ where $j \in\{1, \ldots, h-1\}$ corresponds to a triangle (clique of size three) in $L\left(W_{h}\right)$, while vertex $v^{x, h}$ corresponds to a clique of size two. Let us say that a triangle in $L\left(W_{h}\right)$ is upward pointing if it arises from a vertex in $W_{h}$ whose coordinates have even sum, and downward pointing, otherwise. (We draw this terminology from the planar embeddings of the line graphs of the walls following the example given in Fig. 4.) It is not difficult to see that for every $x \in X_{h}$ and every even $i \in\{0,1, \ldots, h-2\}$, vertex $v^{x, i}$ corresponds to an upward triangle, while odd-indexed vertices may correspond to either upward or downward pointing triangles. It follows that for no index $i \in\{0,1, \ldots, h-1\}$, vertices $v^{x, i}$ and $v^{x, i+1}$ can both correspond to downward pointing triangles. This property ensures that the graph $L\left(W_{h}\right)-S_{x}^{\prime}$ is disconnected, with exactly two components $C^{\prime}$ and $D^{\prime}$ such that for all $v^{x, j} \in S_{x}$, component $C^{\prime}$ contains all vertices of the form $e^{x, j^{-}}$, where $e^{x, j^{-}} \in V\left(L\left(W_{h}\right)\right)$ is the vertex corresponding to the edge in $W_{h}$ joining vertex $v^{x, j}$ with vertex $v^{x, j}-(1,0)$, while component $D^{\prime}$ contains all vertices of the form $e^{x, j^{+}}$, where $e^{x, j^{+}} \in V\left(L\left(W_{h}\right)\right)$ is the vertex corresponding to the edge in $W_{h}$ 
joining vertex $v^{x, j}+(1,0)$ with vertex $v^{x, j}+(2,0)$. Furthermore, since for every vertex $e^{x, j} \in S_{x}^{\prime}$, vertices $e^{x, j^{-}}$and $e^{x, j^{+}}$are both adjacent to $e^{x, j}$ in $L\left(W_{h}\right)$, this also implies, by Lemma 9, that $S_{x}^{\prime}$ is a minimal separator in $L\left(W_{h}\right)$. This completes the proof. Fig. 4(b) shows an example with $h=8$ and $x=(1,1,0,0,1,1,1,1)$. The thick horizontal edges can be used to justify the fact that $C^{\prime}$ and $D^{\prime}$ are $S_{x}^{\prime}$-full components of $L\left(W_{h}\right)-S_{x}^{\prime}$.

In particular, since line graphs of elementary walls are $\left\{\right.$ claw, $K_{4}, C_{4}$, diamond $\}$-free, Proposition 40 implies the following.

Corollary 41. The class of $\left\{\right.$ claw, $K_{4}, C_{4}$, diamond $\}$-free graphs is not tame.

\section{A dichotomy for small forbidden induced subgraphs}

In this section we prove Theorem 7. First we prove a number of propositions, each giving a sufficient condition for a family $\mathcal{F}$ of graphs on at most 4 vertices such that the class of $\mathcal{F}$-free graphs is tame. We start with a lemma simplifying the cases with $P_{3}+P_{1} \in \mathcal{F}$.

Lemma 42. Let $\mathcal{F}$ be a family of graphs such that $P_{3}+P_{1} \in \mathcal{F}$ and let $\mathcal{F}^{\prime}=\left(\mathcal{F} \backslash\left\{P_{3}+P_{1}\right\}\right) \cup\left\{3 P_{1}\right\}$. Then the class of $\mathcal{F}$-free graphs is tame if and only if the class of $\mathcal{F}^{\prime}$-free graphs is tame.

Proof. Let $\mathcal{G}$ and $\mathcal{G}^{\prime}$ be the classes of $\mathcal{F}$-free and $\mathcal{F}^{\prime}$-free graphs, respectively. Clearly, every $\mathcal{F}^{\prime}$-free graph is also $\mathcal{F}$-free (cf. Observation 6), and hence if $\mathcal{G}$ is tame, then so is $\mathcal{G}^{\prime}$. Suppose that $\mathcal{G}^{\prime}$ is tame. By Lemma 16, there exists an integer $k \geqslant 0$ such that $s(G) \leqslant|V(G)|^{k}-1$ for all $G \in \mathcal{G}^{\prime}$. Let $G \in \mathcal{G}$. By Corollary 22 we may assume that $G$ is anticonnected. Since $G$ is anticonnected and $\left(P_{3}+P_{1}\right)$-free, applying Theorem 8 to the complement of $G$ implies that $G$ is either a disjoint union of complete graphs, in which case $s(G) \leqslant 1$, or $G$ is $3 P_{1}$-free, in which case $G \in \mathcal{G}^{\prime}$ and thus $s(G) \leqslant|V(G)|^{k}-1$. It follows that $\mathcal{G}$ is tame.

We now consider various families of forbidden induced subgraphs with at most four vertices. We will also need the following result describing the structure of $\left\{\right.$ claw, $C_{3}+$ $P_{1}$ \}-free graphs. By $S_{3}$ we denote the 6-vertex graph obtained from the 6-cycle with vertices $v_{1}, \ldots, v_{6}$ in cyclic order by adding to it the chords $v_{1} v_{3}, v_{3} v_{5}$, and $v_{5} v_{1}$.

Theorem 43 (Pouzet et al. [43]). The class of $\left\{\right.$ claw, $\left.C_{3}+P_{1}\right\}$-free graphs consists of $S_{3}$, of the induced subgraphs of $L\left(K_{3,3}\right)$, of graphs whose connected components are cycles of length at least 4 or paths, and of the complements of these graphs.

Proposition 44. For every $F \in\left\{4 P_{1}, P_{2}+2 P_{1}, P_{3}+P_{1}\right.$, claw $\}$, the class of $\left\{F, C_{3}+\right.$ $\left.P_{1}\right\}$-free graphs is tame.

Proof. i) The class of $\left\{4 P_{1}, C_{3}+P_{1}\right\}$-free graphs is a subclass of the class of $\left\{P_{2}+4 P_{1}\right.$, $\left.C_{3}+P_{2}\right\}$-free graphs, which is tame by Theorem 5 .

ii) The class of $\left\{P_{2}+2 P_{1}, C_{3}+P_{1}\right\}$-free graphs is a subclass of the class of $\left\{P_{2}+2 P_{1}\right.$, $\left.C_{3}+P_{2}\right\}$-free graphs, which is tame by Theorem 5 . 
iii) By Lemma 42 , it suffices to show that the class of $\left\{3 P_{1}, C_{3}+P_{1}\right\}$-free graphs is tame. This follows from part i) of the proposition.

iv) By Corollaries 19 and 22, it suffices to prove that the class of connected and anticonnected \{claw, $\left.C_{3}+P_{1}\right\}$-free graphs is tame. Let $G$ be a connected and anticonnected $\left\{\right.$ claw, $\left.C_{3}+P_{1}\right\}$-free graph with at least 10 vertices. By Theorem 43 , $G$ is either a path or a cycle, or the complement of a path or of a cycle. If $G$ is a path or a cycle, then all its minimal separators have size 1 or 2 , respectively. If the complement of $G$ is of a path or a cycle, then $G$ is $2 P_{2}$-free, and hence has at most $|V(G)|$ minimal separators by Lemma 12 . Thus, in either case the number of minimal separators of $G$ is polynomially bounded.

Proposition 45. The class of $\left\{P_{3}+P_{1}, C_{4}\right\}$-free graphs is tame.

Proof. Immediate from Lemma 42 and the fact that the class of $\left\{3 P_{1}, C_{4}\right\}$-free graphs is tame, which follows from Corollary 32.

Proposition 46. For every $F \in\left\{4 P_{1}, P_{2}+2 P_{1}, P_{3}+P_{1}\right\}$, the class of $\left\{F, K_{4}\right\}$-free graphs is tame.

Proof. i) By Ramsey's theorem, the class of $\left\{4 P_{1}, K_{4}\right\}$-free graph consists of finitely many graphs, so it is tame.

ii) The class of $\left\{P_{2}+2 P_{1}, K_{4}\right\}$-free graphs is a subclass of the class of $\left\{P_{2}+2 P_{1}\right.$, $\left.K_{4}+P_{2}\right\}$-free graphs, which is tame by Theorem 5 .

iii) By Lemma 42, it suffices to show that the class of $\left\{3 P_{1}, K_{4}\right\}$-free graphs is tame. This follows from part i) of the proposition.

In the proofs of Proposition 48 and Theorem 7, we will also need the following result, which is a consequence of more general results due to Nikolopoulos and Palios [39] and Pedrotti and de Mello [42].

Theorem 47. The class of $P_{4}$-free graphs is tame.

Proposition 48. For every $F \in\left\{4 P_{1}, P_{2}+2 P_{1}, P_{3}+P_{1}\right.$, claw $\}$, the class of $\{F$, paw $\}$-free graphs is tame.

Proof. Let $G$ be an $\{F$, paw $\}$-free graph. By Corollary 19, we may assume that $G$ is connected. Since $G$ is paw-free, Theorem 8 implies that $G$ is either $C_{3}$-free, or complete multipartite. If $G$ is complete multipartite, then $G$ is $P_{4}$-free, and thus has a polynomially bounded number of minimal separators by Theorem 47. Suppose now that $G$ is $C_{3}$-free. If $F \in\left\{4 P_{1}, P_{2}+2 P_{1}, P_{3}+P_{1}\right\}$, then using the fact that $G$ is $K_{4}$-free, Proposition 46 implies that $G$ has a polynomially bounded number of minimal separators. If $F$ is the claw, then $G$ is a $\left\{\right.$ claw, $\left.C_{3}\right\}$-free graph, hence $G$ is a path or a cycle, and all its minimal separators have size 1 or 2 , respectively. In either case, $G$ has a polynomially bounded number of minimal separators. Thus, the class of $\{F$, paw $\}$-free graphs is tame. 
We now have everything ready to prove Theorem 7 .

Theorem 7 (restated). For every family $\mathcal{F}$ of graphs with at most 4 vertices, the following statements are equivalent.

1. The class of $\mathcal{F}$-free graphs is tame.

2. The class of $\mathcal{F}$-free graphs does not contain any of the following graph classes: the class of $\left\{C_{3}, C_{4}\right\}$-free graphs, the class of $\left\{3 P_{1}\right.$, diamond $\}$-free graphs, and the class of $\left\{\right.$ claw, $K_{4}, C_{4}$, diamond $\}$-free graphs.

3. $\mathcal{F} \unlhd \mathcal{F}^{\prime}$ for at least one of the following families $\mathcal{F}^{\prime}$ :

i) $\mathcal{F}^{\prime}=\left\{P_{4}\right\}$,

ii) $\mathcal{F}^{\prime}=\left\{2 P_{2}\right\}$,

iii) $\mathcal{F}^{\prime}=\{F$, paw $\}$ for some $F \in\left\{4 P_{1}, P_{2}+2 P_{1}, P_{3}+P_{1}\right.$, claw $\}$,

iv) $\mathcal{F}^{\prime}=\left\{F, C_{3}+P_{1}\right\}$ for some $F \in\left\{4 P_{1}, P_{2}+2 P_{1}, P_{3}+P_{1}\right.$, claw $\}$,

v) $\mathcal{F}^{\prime}=\left\{F, K_{4}\right\}$ for some $F \in\left\{4 P_{1}, P_{2}+2 P_{1}, P_{3}+P_{1}\right\}$,

vi) $\mathcal{F}^{\prime}=\left\{F, C_{4}\right\}$ for some $F \in\left\{4 P_{1}, P_{2}+2 P_{1}, P_{3}+P_{1}\right\}$.

Proof. Let $\mathcal{F}$ be a family of graphs on at most 4 vertices.

Suppose first that the class of $\mathcal{F}$-free graphs is tame and, for a contradiction, that the class of $\mathcal{F}$-free graphs contains the class of $\mathcal{F}^{\prime}$-free graphs for some $\mathcal{F}^{\prime} \in\left\{\left\{C_{3}, C_{4}\right\},\left\{3 P_{1}\right.\right.$, diamond $\}$, \{claw, $K_{4}, C_{4}$, diamond $\}$. If $\mathcal{F}^{\prime}=\left\{C_{3}, C_{4}\right\}$, then the class of $\mathcal{F}^{\prime}$-free graphs is not tame by Proposition 38. If $\mathcal{F}^{\prime}=\left\{3 P_{1}\right.$, diamond $\}$, then the class of $\mathcal{F}^{\prime}$-free graphs is not tame by Corollary 37. If $\mathcal{F}^{\prime}=\left\{\right.$ claw, $K_{4}, C_{4}$, diamond $\}$, then the class of $\mathcal{F}^{\prime}$-free graphs is not tame by Corollary 41. It follows by Observation 14 that the class of $\mathcal{F}$-free graphs is not tame, a contradiction. Thus, the first statement implies the second one.

Suppose now that for all $\mathcal{F}^{\prime} \in\left\{\left\{C_{3}, C_{4}\right\},\left\{3 P_{1}\right.\right.$, diamond $\}$, \{claw, $K_{4}, C_{4}$, diamond $\}$, the class of $\mathcal{F}$-free graphs does not contain the class of $\mathcal{F}^{\prime}$-free graphs. We want to prove that $\mathcal{F} \unlhd \mathcal{F}^{\prime}$ where $\mathcal{F}^{\prime}$ satisfies one of the following: $\mathcal{F}^{\prime}=\left\{P_{4}\right\}, \mathcal{F}^{\prime}=\left\{2 P_{2}\right\}, \mathcal{F}^{\prime}=\{F$, paw $\}$ for some $F \in\left\{4 P_{1}, P_{2}+2 P_{1}, P_{3}+P_{1}\right.$, claw $\}, \mathcal{F}^{\prime}=\left\{F, C_{3}+P_{1}\right\}$ for some $F \in\left\{4 P_{1}\right.$, $P_{2}+2 P_{1}, P_{3}+P_{1}$, claw $\}, \mathcal{F}^{\prime}=\left\{F, K_{4}\right\}$ for some $F \in\left\{4 P_{1}, P_{2}+2 P_{1}, P_{3}+P_{1}\right\}$, or $\mathcal{F}^{\prime}=\left\{F, C_{4}\right\}$ for some $F \in\left\{4 P_{1}, P_{2}+2 P_{1}, P_{3}+P_{1}\right\}$.

If some graph $F \in \mathcal{F}$ is an induced subgraph of $2 P_{2}$ or of $P_{4}$, then $\mathcal{F} \unlhd \mathcal{F}^{\prime}$ for $\mathcal{F}^{\prime}=\left\{2 P_{2}\right\}$ or $\mathcal{F}^{\prime}=\left\{P_{4}\right\}$. Thus, from now on we assume that no graph $F \in \mathcal{F}$ is an induced subgraph of either $2 P_{2}$ or $P_{4}$. Let $A=\left\{C_{3}, C_{4}, C_{3}+P_{1}\right.$, paw, diamond, $\left.K_{4}\right\}$ and $B=\left\{3 P_{1}, 4 P_{1}, P_{2}+2 P_{1}, P_{3}+P_{1}\right.$, claw $\}$. Note that every member of $B$ contains $3 P_{1}$ as an induced subgraph, and similarly every member of $A$ contains $C_{3}$ or $C_{4}$ as an induced subgraph. Since every graph in $\mathcal{F}$ has at most 4 vertices and is not an induced subgraph of either $2 P_{2}$ or $P_{4}$, we infer that $\mathcal{F} \subseteq A \cup B$. Let $A^{\prime}=A \backslash\{$ diamond $\}$. If $\mathcal{F} \cap A^{\prime}=\emptyset$, then $\mathcal{F} \subseteq\{$ diamond $\} \cup B$ and the class of $\mathcal{F}$-free graphs contains the class of $\left\{3 P_{1}\right.$, diamond $\}$-free graphs, a contradiction. It follows that $\mathcal{F} \cap A^{\prime} \neq \emptyset$. If $\mathcal{F} \cap B=\emptyset$, then $\mathcal{F} \subseteq A$ and the class of $\mathcal{F}$-free graphs contains the class of $\left\{C_{3}, C_{4}\right\}$-free graphs, a 
contradiction. Therefore, $\mathcal{F} \cap B \neq \emptyset$. If $\mathcal{F} \cap\left\{C_{3}, C_{3}+P_{1}\right.$, paw $\} \neq \emptyset$, then the fact that $\mathcal{F} \cap B \neq \emptyset$ implies that $\mathcal{F} \unlhd \mathcal{F}^{\prime}$ where $\mathcal{F}^{\prime}=\{F$, paw $\}$ or $\mathcal{F}^{\prime}=\left\{F, C_{3}+P_{1}\right\}$ for some $F \in\left\{4 P_{1}, P_{2}+2 P_{1}, P_{3}+P_{1}\right.$, claw $\}$. Assume now that $\mathcal{F} \cap\left\{C_{3}, C_{3}+P_{1}\right.$, paw $\}=\emptyset$. As $\mathcal{F} \cap A^{\prime} \neq \emptyset$, it follows that $\mathcal{F} \cap\left\{C_{4}, K_{4}\right\} \neq \emptyset$. Let $B^{\prime}=B \backslash\{$ claw $\}$. If $\mathcal{F} \cap B^{\prime}=\emptyset$, then $\mathcal{F} \subseteq\left\{\right.$ claw, $C_{4}, K_{4}$, diamond $\}$ and the class of $\mathcal{F}$-free graphs contains the class of \{claw, $K_{4}, C_{4}$, diamond $\}$-free graphs, a contradiction. It follows that $\mathcal{F} \cap B^{\prime} \neq \emptyset$. Therefore, using that $\mathcal{F} \cap\left\{C_{4}, K_{4}\right\} \neq \emptyset$, we infer that $\mathcal{F} \unlhd \mathcal{F}^{\prime}$ where $\mathcal{F}^{\prime}=\left\{F, C_{4}\right\}$ or $\mathcal{F}^{\prime}=\left\{F, K_{4}\right\}$ for some $F \in\left\{4 P_{1}, P_{2}+2 P_{1}, P_{3}+P_{1}\right\}$. Thus, the second statement implies the third one.

Finally, suppose that $\mathcal{F} \unlhd \mathcal{F}^{\prime}$ where $\mathcal{F}^{\prime}$ satisfies one of the following: $\mathcal{F}^{\prime}=\left\{P_{4}\right\}$, $\mathcal{F}^{\prime}=\left\{2 P_{2}\right\}, \mathcal{F}^{\prime}=\{F$, paw $\}$ for some $F \in\left\{4 P_{1}, P_{2}+2 P_{1}, P_{3}+P_{1}\right.$, claw $\}, \mathcal{F}^{\prime}=\{F$, $\left.C_{3}+P_{1}\right\}$ for some $F \in\left\{4 P_{1}, P_{2}+2 P_{1}, P_{3}+P_{1}\right.$, claw $\}, \mathcal{F}^{\prime}=\left\{F, K_{4}\right\}$ for some $F \in\left\{4 P_{1}\right.$, $\left.P_{2}+2 P_{1}, P_{3}+P_{1}\right\}$, or $\mathcal{F}^{\prime}=\left\{F, C_{4}\right\}$ for some $F \in\left\{4 P_{1}, P_{2}+2 P_{1}, P_{3}+P_{1}\right\}$. Note that the class of $\mathcal{F}$-free graphs is contained in the class of $\mathcal{F}^{\prime}$-free graphs, hence by Observation 14 it suffices to show that the class of $\mathcal{F}^{\prime}$-free graphs is tame. If $\mathcal{F}^{\prime}=\left\{P_{4}\right\}$ or $\mathcal{F}^{\prime}=\left\{2 P_{2}\right\}$, then the class of $\mathcal{F}^{\prime}$-free graphs is tame by Theorem 47 and Corollary 13, respectively. If $\mathcal{F}^{\prime}=\{F$, paw $\}$ for some $F \in\left\{4 P_{1}, P_{2}+2 P_{1}, P_{3}+P_{1}\right.$, claw $\}$, then the class of $\mathcal{F}^{\prime}$-free graphs is tame by Proposition 48. If $\mathcal{F}^{\prime}=\left\{F, C_{3}+P_{1}\right\}$ for some $F \in\left\{4 P_{1}, P_{2}+2 P_{1}\right.$, $P_{3}+P_{1}$, claw $\}$, then the class of $\mathcal{F}^{\prime}$-free graphs is tame by Proposition 44. If $\mathcal{F}^{\prime}=\{F$, $\left.K_{4}\right\}$ for some $F \in\left\{4 P_{1}, P_{2}+2 P_{1}, P_{3}+P_{1}\right\}$, then the class of $\mathcal{F}^{\prime}$-free graphs is tame by Proposition 46. Finally, if $\mathcal{F}^{\prime}=\left\{F, C_{4}\right\}$ for some $F \in\left\{4 P_{1}, P_{2}+2 P_{1}, P_{3}+P_{1}\right\}$, then the class of $\mathcal{F}^{\prime}$-free graphs is tame by Corollary 32 (if $F=4 P_{1}$ ), by Proposition 45 (if $F=P_{3}+P_{1}$ ), or by Theorem 4 (if $F=P_{2}+2 P_{1}$ ). This shows that the third statement implies the first one and completes the proof.

\section{Open problems}

In this work we considered classes of graphs with polynomially many minimal separators and examined them from various points of view. Constructions of graphs with exponentially many minimal separators and the newly identified families of tame graph classes led to a complete classification of the tame graph classes within the family of graph classes defined by forbidden induced subgraphs with at most four vertices. Several of the results given here are not restricted to forbidden induced subgraphs of at most four vertices, and we hope they might prove useful for developing further results on minimal separators.

In conclusion, we pose the following open problems:

1. It is not difficult to see that given a graph $H$, the class of $H$-free graphs is tame if and only if $H$ is an induced subgraph of either $P_{4}$ or of $2 P_{2}$. Which graph classes defined by two forbidden induced subgraphs are tame? More generally, which graph classes defined by finitely many forbidden induced subgraphs are tame?

2. Which graph classes have a linear number of minimal separators?

3. We are not aware of any hereditary tame graph class in which the $k$-coloring problem 
(for some fixed $k \geqslant 3$ ) is known to be NP-hard. Are the $k$-coloring problems polynomial-time solvable in every hereditary tame graph class?

4. Is every hereditary tame graph class $\chi$-bounded? That is, does every hereditary tame graph class $\mathcal{G}$ admit a function $f$ such that $\chi(G) \leqslant f(\omega(G))$ for all $G \in$ $\mathcal{G}$ (where $\omega(G)$ and $\chi(G)$ denote the clique number and chromatic number of $G$, respectively)? A positive answer to this question would give a partial solution to the question of Thomassé et al. from [52] asking whether every hereditary graph class $\mathcal{G}$ in which the maximum independent set problem is polynomial-time solvable is $\chi$-bounded.

\section{Acknowledgements}

The authors are grateful to the anonymous reviewers for their helpful remarks.

\section{References}

[1] V. E. Alekseev. On the number of maximal independent sets in graphs from hereditary classes. Combinatorial-algebraic methods in discrete optimization, University of Nizhny Novgorod, pages 5-8, 1991. (in Russian).

[2] J. Backer. Separator orders in interval, cocomparability, and AT-free graphs. Discrete Appl. Math., 159(8):717-726, 2011.

[3] E. Balas and C. S. Yu. On graphs with polynomially solvable maximum-weight clique problem. Networks, 19(2):247-253, 1989.

[4] E. Berger, K. Choromanski, M. Chudnovsky, J. Fox, M. Loebl, A. Scott, P. Seymour, and S. Thomassé. Tournaments and colouring. J. Combin. Theory Ser. B, 103(1):1-20, 2013.

[5] A. Berry and J.-P. Bordat. Separability generalizes Dirac's theorem. Discrete Appl. Math., 84(1-3):43-53, 1998.

[6] H. L. Bodlaender, T. Kloks, and D. Kratsch. Treewidth and pathwidth of permutation graphs. SIAM J. Discrete Math., 8(4):606-616, 1995.

[7] H. L. Bodlaender and A. M. C. A. Koster. Safe separators for treewidth. Discrete Math., 306(3):337-350, 2006.

[8] H. L. Bodlaender and A. M. C. A. Koster. Treewidth computations. I. Upper bounds. Inform. and Comput., 208(3):259-275, 2010.

[9] H. L. Bodlaender and U. Rotics. Computing the treewidth and the minimum fill-in with the modular decomposition. Algorithmica, 36(4):375-408, 2003.

[10] V. Boncompagni, I. Penev, and K. Vušković. Clique-cutsets beyond chordal graphs. J. Graph Theory, 91(2):192-246, 2019.

[11] V. Bouchitté and I. Todinca. Treewidth and minimum fill-in: grouping the minimal separators. SIAM J. Comput., 31(1):212-232, 2001. 
[12] V. Bouchitté and I. Todinca. Listing all potential maximal cliques of a graph. Theoret. Comput. Sci., 276(1-2):17-32, 2002.

[13] A. Brandstädt, J. Engelfriet, H.-O. Le, and V. V. Lozin. Clique-width for 4-vertex forbidden subgraphs. Theory Comput. Syst., 39(4):561-590, 2006.

[14] R. C. Brewster, P. Hell, and R. Rizzi. Oriented star packings. J. Combin. Theory Ser. B, 98(3):558-576, 2008.

[15] G. J. Chang, A. J. J. Kloks, J. Liu, and S.-L. Peng. The PIGs Full Monty-a floor show of minimal separators. In STACS 2005, volume 3404 of Lecture Notes in Comput. Sci., pages 521-532. Springer, Berlin, 2005.

[16] N. Chiarelli and M. Milanič. Linear separation of connected dominating sets in graphs. Ars Math. Contemp., 16(2):487-525, 2019.

[17] D. Coudert, G. Ducoffe, and N. Nisse. To approximate treewidth, use treelength! SIAM J. Discrete Math., 30(3):1424-1436, 2016.

[18] K. K. Dabrowski, M. Johnson, G. Paesani, D. Paulusma, and V. Zamaraev. On the price of independence for vertex cover, feedback vertex set and odd cycle transversal. In 43rd International Symposium on Mathematical Foundations of Computer Science, volume 117 of LIPIcs. Leibniz Int. Proc. Inform., pages Art. No. 63, 15. Schloss Dagstuhl. Leibniz-Zent. Inform., Wadern, 2018.

[19] K. K. Dabrowski, M. Johnson, and D. Paulusma. Clique-width for hereditary graph classes. In Surveys in combinatorics 2019, volume 456 of London Math. Soc. Lecture Note Ser., pages 1-56. Cambridge Univ. Press, Cambridge, 2019.

[20] J. S. Deogun, T. Kloks, D. Kratsch, and H. Müller. On the vertex ranking problem for trapezoid, circular-arc and other graphs. Discrete Appl. Math., 98(1-2):39-63, 1999.

[21] G. A. Dirac. On rigid circuit graphs. Abh. Math. Sem. Univ. Hamburg, 25:71-76, 1961.

[22] F. V. Fomin, I. Todinca, and Y. Villanger. Large induced subgraphs via triangulations and CMSO. SIAM J. Comput., 44(1):54-87, 2015.

[23] F. V. Fomin and Y. Villanger. Finding induced subgraphs via minimal triangulations. In STACS 2010: 27th International Symposium on Theoretical Aspects of Computer Science, volume 5 of LIPIcs. Leibniz Int. Proc. Inform., pages 383-394. Schloss Dagstuhl. Leibniz-Zent. Inform., Wadern, 2010.

[24] P. A. Golovach, M. Johnson, D. Paulusma, and J. Song. A survey on the computational complexity of coloring graphs with forbidden subgraphs. J. Graph Theory, 84(4):331-363, 2017.

[25] M. C. Golumbic. Algorithmic Graph Theory and Perfect Graphs, volume 57 of Annals of Discrete Mathematics. Elsevier Science B.V., Amsterdam, second edition, 2004.

[26] T. R. Hartinger, M. Johnson, M. Milanič, and D. Paulusma. The price of connectivity for cycle transversals. European J. Combin., 58:203-224, 2016. 
[27] P. Heggernes. Minimal triangulations of graphs: a survey. Discrete Math., 306(3):297-317, 2006.

[28] P. Hell and J. Nešetřil. On the complexity of H-coloring. J. Combin. Theory Ser. B, 48(1):92-110, 1990.

[29] T. Kloks. Treewidth, volume 842 of Lecture Notes in Computer Science. Springer-Verlag, Berlin, 1994.

[30] T. Kloks. Treewidth of circle graphs. International Journal of Foundations of Computer Science, 7(02):111-120, 1996.

[31] T. Kloks, H. Bodlaender, H. Müller, and D. Kratsch. Computing treewidth and minimum fill-in: all you need are the minimal separators. In Algorithms - ESA '93 (Bad Honnef, 1993), volume 726 of Lecture Notes in Comput. Sci., pages 260-271. Springer, Berlin, 1993.

[32] T. Kloks, D. Kratsch, and J. Spinrad. On treewidth and minimum fill-in of asteroidal triple-free graphs. Theoret. Comput. Sci., 175(2):309-335, 1997.

[33] T. Kloks, D. Kratsch, and C. K. Wong. Minimum fill-in on circle and circular-arc graphs. J. Algorithms, 28(2):272-289, 1998.

[34] E. Köhler. Graphs without asteroidal triples. PhD thesis, Technische Universität Berlin, 1999.

[35] M. Loebl and S. Poljak. Efficient subgraph packing. J. Combin. Theory Ser. B, 59(1):106-121, 1993.

[36] D. S. Malyshev. A complexity dichotomy and a new boundary class for the dominating set problem. J. Comb. Optim., 32(1):226-243, 2016.

[37] M. Milanič and N. Pivač. Minimal separators in graph classes defined by small forbidden induced subgraphs. In Graph-Theoretic Concepts in Computer Science, volume 11789 of Lecture Notes in Comput. Sci., pages 379-391. 2019.

[38] P. Montealegre and I. Todinca. On distance- $d$ independent set and other problems in graphs with "few" minimal separators. In Graph-Theoretic Concepts in Computer Science, volume 9941 of Lecture Notes in Comput. Sci., pages 183-194. Springer, Berlin, 2016.

[39] S. D. Nikolopoulos and L. Palios. Minimal separators in $P_{4}$-sparse graphs. Discrete Math., 306(3):381-392, 2006.

[40] S. Olariu. Paw-free graphs. Inform. Process. Lett., 28(1):53-54, 1988.

[41] A. Parra and P. Scheffler. Characterizations and algorithmic applications of chordal graph embeddings. Discrete Appl. Math., 79(1-3):171-188, 1997.

[42] V. Pedrotti and C. P. de Mello. Minimal separators in extended $P_{4}$-laden graphs. Discrete Appl. Math., 160(18):2769-2777, 2012.

[43] M. Pouzet, H. Si Kaddour, and N. Trotignon. Claw-freeness, 3-homogeneous subsets of a graph and a reconstruction problem. Contrib. Discrete Math., 6(1):86-97, 2011. 
[44] E. Prisner. Graphs with few cliques. In Graph theory, combinatorics, and algorithms, Vol. 1, 2 (Kalamazoo, MI, 1992), Wiley-Intersci. Publ., pages 945-956. Wiley, New York.

[45] F. P. Ramsey. On a Problem of Formal Logic. Proc. London Math. Soc. (2), 30(4):264-286, 1929.

[46] D. J. Rose, R. E. Tarjan, and G. S. Lueker. Algorithmic aspects of vertex elimination on graphs. SIAM J. Comput., 5(2):266-283, 1976.

[47] P. Schweitzer. Towards an isomorphism dichotomy for hereditary graph classes. Theory Comput. Syst., 61(4):1084-1127, 2017.

[48] K. Skodinis. Efficient analysis of graphs with small minimal separators. In Graph-Theoretic Concepts in Computer Science, volume 1665 of Lecture Notes in Comput. Sci., pages 155-166. Springer, Berlin, 1999.

[49] K. Suchan. Minimal Separators in Intersection Graphs. Master's thesis, Akademia Górniczo-Hutnicza im. Stanisława Staszica w Krakowie, 2003.

[50] H. Tamaki. Computing treewidth via exact and heuristic lists of minimal separators. In I. Kotsireas, P. M. Pardalos, K. E. Parsopoulos, D. Souravlias, and A. Tsokas, editors, Analysis of Experimental Algorithms - Special Event, SEA 2019, Kalamata, Greece, June 24-29, 2019, Revised Selected Papers, volume 11544 of Lecture Notes in Computer Science, pages 219-236. Springer, 2019.

[51] R. E. Tarjan. Decomposition by clique separators. Discrete Math., 55(2):221-232, 1985.

[52] S. Thomassé, N. Trotignon, and K. Vušković. A polynomial Turing-kernel for weighted independent set in bull-free graphs. Algorithmica, 77(3):619-641, 2017.

[53] S. Wagon. A bound on the chromatic number of graphs without certain induced subgraphs. J. Combin. Theory Ser. B, 29(3):345-346, 1980. 